\title{
Expressiveness and Performance of Full-Text Search Languages
}

\author{
Chavdar Botev \\ Cornell University \\ cbotev@cs.cornell.edu
}

\author{
Sihem Amer-Yahia \\ AT\&T Labs-Research \\ sihem@research.att.com
}

\author{
Jayavel Shanmugasundaram \\ Cornell University \\ jai@cs.cornell.edu
}

\begin{abstract}
We study the expressiveness and performance of full-text search languages. Our main motivation is to provide a formal basis for comparing such languages and to develop a model for full-text search that can be tightly integrated with structured search. We develop a formal model for full-text search based on the positions of tokens (words) in the input text, and develop a full-text calculus (FTC) and a full-text algebra (FTA) with equivalent expressive power. This suggests a notion of completeness for full-text search languages and can be used as a basis for a study of their expressiveness. We show that existing full-text languages are incomplete and develop COMP, a complete full-text search language. We also identify practical subsets of COMP that are more powerful than existing languages, develop efficient query evaluation algorithms for these subsets, and study experimentally their performance.
\end{abstract}

\section{Introduction}

There has been a lot of interest in full-text search over flat files [3, 31], relational databases [4, 22], and more recently, XML documents $[2,6,15,18,21,33]$. The expressiveness of such languages ranges from simple Boolean search to phrase matching to general proximity search with distance predicates. Unfortunately, there is no existing work that systematically compares these different full-text search languages, either from an expressiveness or a performance point of view; we believe that this void is mainly due to the lack of a powerful formal model for full-text search. This also makes it difficult to seamlessly integrate with structured search, which is usually based on the formal underpinnings of the relational model. In this paper, we attempt to lay down the formal foundations for full-text search languages, and to compare the expressiveness and performance of different languages.

Our first contribution (Section 2) is the development of a formal model for full-text search languages. At an abstract level, such languages require the ability to manipulate individual tokens (or words) and their positions in the input text and return the nodes (e.g., documents, tuples, or XML elements) that satisfy the full-text search condition. We thus define a formal model based on the positions of tokens in the input text. Based on this model, we define a notion of completeness and develop an associated full-text calculus (FTC) based on first-order logic and a full-text algebra (FTA) based on the relational algebra. We also show how the FTC and FTA can be extended to capture the notion of scores, such as scores computed using TF-IDF [3, 28].

Our second contribution (Section 4) is to show that existing languages are incomplete. We thus propose COMP, a new complete full-text search language based on the FTC. COMP naturally generalizes existing Boolean full-text search languages and is able to express primitives such as order specifications between words, paragraph scoping and word distance.

Our third distribution is the design of a scoring framework that can be used within our full-text search model. The scoring framework does not mandate a fixed scoring method but allows the use of large class of 
existing scoring methods. In Section 3, we describe the framework and show how it can be used with two of the most popular scoring methods: TF-IDF [28] and probabilistic scoring [19, 38]

Our fourth contribution (Sections 5 and 6 ) is the identification of practical subsets of COMP that are significantly more powerful than existing full-text search languages, but which can still be evaluated in a linear scan over inverted list data structures, which are commonly used in full-text search. We also experimentally evaluate our proposed algorithms using real and synthetic data sets.

\section{Full-Text Model, Full-Text Calculus and Algebra}

At its core, a full-text search specification has two components: (1) the search context, which specifies the set of context nodes (e.g., document corpus in an IR system, tuples in a relational database or elements in XML documents) over which the full-text search is to be performed and, (2) the full-text condition, which specifies the condition that should be evaluated on each context node. Only the context nodes that satisfy the full-text condition qualify as answers. As an illustration, consider the following example from the XQuery Full-Text Use Cases Document [35].

Example 1 (Use Case 10.4): Given an XML document that contains book and article elements, find the book elements containing the "efficient" and the phrase "task completion" in that order with at most 10 intervening tokens.

In this example, the context nodes are book elements (and not articles), and the full-text search condition is: contains the keyword "efficient" and the phrase "task completion" in that order with at most 10 intervening tokens. Note that the search condition includes multiple primitives: Boolean AND, phrase matching, order specifications, and distance predicates.

Thus, in order to specify a full-text search query, we need (1) a language to specify the context nodes and, (2) a language to specify the search condition (the full-text search language). For (1), we can use SQL in the case of relational data, XQuery in the case of XML documents, or simply the entire document collection in the case of traditional IR systems. Since SQL and XQuery have well-defined formal semantics, we focus on the full-text search language. We first present our model, before discussing the FTC, the FTA, and scoring issues.

\subsection{Full-Text Model}

We first introduce two aspects of our model: a model for context nodes, and our requirement for completeness.

\subsubsection{Modeling Context Nodes}

Existing models for context nodes are insufficient for expressive full-text search. For instance, the XQuery data model for the book element in Figure 1 treats all the text under an element as a single text node (ignore the numbers in parentheses for now). This model is enough to identify sub-strings in the text and evaluate queries such as find author nodes containing 'Elina'. However, it is insufficient to answer queries such as find books that contain the tokens 'usability' and 'testing' with 3 intervening tokens. Most IR models solve part of this problem by tokenizing the input text, and representing each token separately. Thus, in our example, the text in the context node would be modeled as the "bag of words" $\{$ book, id, 1000, author, Elina, Rose, ...\}. However, this model still cannot capture the distance between 


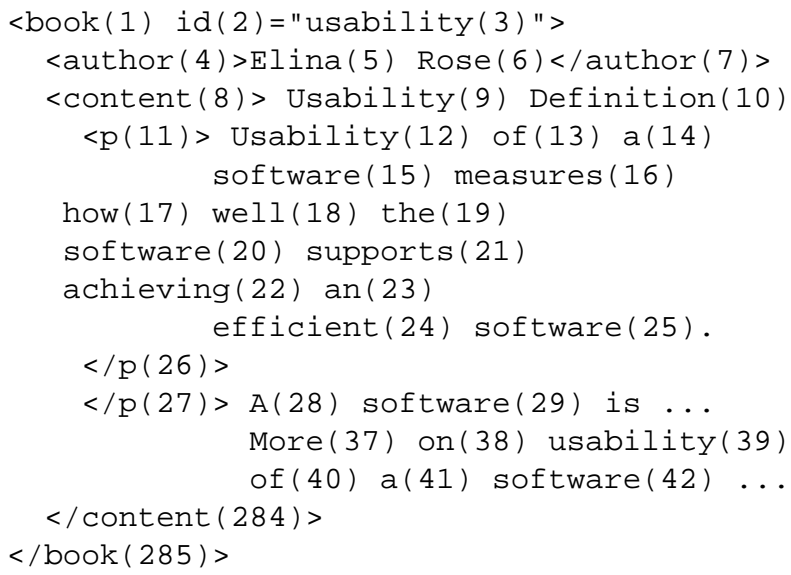

Figure 1: Positions Example

tokens (some IR languages, however, do support limited forms of distance predicates; see Section 4.2 for more details).

In this paper, we explicitly model the position of a token in a context node. We argue that this model, although simple, is powerful enough to capture the semantics of existing full-text search languages. Further, it serves as the formal basis for defining position-based predicates such as proximity distance and order predicates. In Figure 1, we have used a simple numeric position (within parenthesis) for each token. Our proposed model, however, does not dictate any specific implementation of positions and is extensible with respect to the set of predicates. More expressive positions that capture the notions of lines, sentences and paragraphs can be used, and this will enable more sophisticated predicates on positions.

We now define our formal model. $\mathcal{N}$ is the set of context nodes, $\mathcal{P}$ is the set of positions, and $\mathcal{T}$ is the set of tokens. The function Positions $: \mathcal{N} \rightarrow 2^{\mathcal{P}}$ maps a context node to the set of positions in the context node. The function Token $: \mathcal{P} \rightarrow \mathcal{T}$ maps each position to the token stored at that position. In the example in Figure 1, if the context node is denoted by $n$, then Positions $(n)=\{1,2, \ldots, 28\}$, Token $(1)=$ book, $\operatorname{Token}(2)=i d$, and so on.

\subsubsection{Requirement for Completeness}

The full-text search language should be at least as expressive as first-order logic formulae specified over the positions of tokens in a context node.

The above requirement identifies tokens and their positions as the fundamental units in a full-text search language, and essentially describes a notion of completeness similar to that of relational completeness [13] based on first-order logic. We note that other notions of completeness can certainly be defined based on higher-order logics, but as we shall soon see, defining completeness in terms of first-order logic allows for both efficient evaluation and tight integration with the relational model. One other issue to note in the above requirement is that each context node is considered separately, i.e., a full-text search condition does not span multiple context nodes or documents. This is in keeping with the semantics of existing full-text languages, and while other extensions are certainly possible, we do not consider them here. 


\subsection{Full-Text Calculus}

The full-text calculus defines the following predicates to model basic full-text primitives.

- SearchContext(node) is true iff node $\in \mathcal{N}$ (recall that $\mathcal{N}$ is the set of context nodes).

- hasPos(node, pos) is true iff pos $\in$ Positions(node). This predicate explicitly captures the notion of positions in an XML node.

- hasToken $(p o s, t o k)$ is true iff $t o k=$ Token(pos). This predicate captures the relationship between tokens and the positions in which they occur.

A full-text language may also wish to specify an additional set of position-based predicates, Preds, depending on user needs. The calculus is general enough to support arbitrary position-based predicates. Specifically, given a set VarPos of position variables, and a set Consts of constants, the calculus can support any predicate of the form: $\operatorname{pred}\left(p_{1}, \ldots, p_{m}, c_{1}, \ldots, c_{r}\right)$, where $p_{1}, \ldots p_{m} \in \operatorname{VarPos}$ and $c_{1}, \ldots, c_{r} \in$

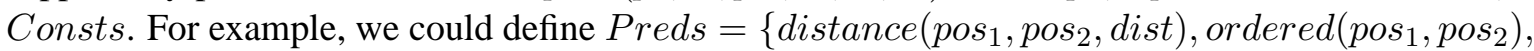
samepara $\left(\operatorname{pos}_{1}, \operatorname{pos}_{2}\right)$, diffpos $\left.\left(\operatorname{pos}_{1}, \operatorname{pos}_{2}\right)\right\}$. Here, distance $\left(\operatorname{pos}_{1}, \operatorname{pos}_{2}\right.$, dist $)$ returns true iff there are at most dist intervening tokens between $\operatorname{pos}_{1}$ and $\operatorname{pos}_{2}$; $\operatorname{ordered}\left(\operatorname{pos}_{1}, \operatorname{pos}_{2}\right)$ is true iff $\operatorname{pos}_{1}$ occurs before $\operatorname{pos}_{2}$; samepara $\left(\operatorname{pos}_{1}, \operatorname{pos}_{2}\right)$ is true iff $\operatorname{pos}_{1}$ is in the same paragraph as $\operatorname{pos}_{2}$; diffpos $\left(\operatorname{pos}_{1}, \operatorname{pos}_{2}\right)$ is true iff $\operatorname{pos}_{1}$ and $\operatorname{pos}_{2}$ are different positions.

\subsubsection{Full-Text Calculus Queries}

A full-text calculus query is of the form: $\{$ node $\mid$ SearchContext(node) $\wedge$ QueryExpr(node) $\}$. Intuitively, the query returns nodes that are in the search context, and that satisfy QueryExpr(node). QueryExpr(node), hereafter called the query expression, is a first-order logic expression that specifies the full-text search condition. node is the only free variable in the query expression. The structure of the query expression is recursively defined as follows.

- hasPos $\left(\right.$ node, $\left._{\text {pos }}\right)$ is a query expression where node is the free variable and $\operatorname{pos}_{i} \in \operatorname{VarPos}$.

- hasToken $\left(\operatorname{pos}_{i}\right.$, tok) is a query expression, where $\operatorname{pos}_{i} \in \operatorname{VarPos}$ and tok $\in$ Consts.

- $\operatorname{pred}\left(\operatorname{pos}_{1}, \ldots, \operatorname{pos}_{m}, c_{1}, \ldots, c_{r}\right)$ is a query expression, where pred $\in \operatorname{Preds}, \operatorname{pos}_{i} \in \operatorname{VarPos}$ and $c_{j} \in$ Consts.

- If $q e_{1}$ and $q e_{2}$ are query expressions, $\neg q e_{1}, q e_{1} \wedge q e_{2}$, and $q e_{1} \vee q e_{2}$ are query expressions.

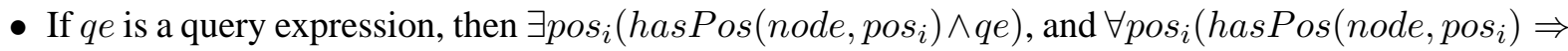
$q e)$ are query expressions, where $\operatorname{pos}_{i} \in \operatorname{VarPos}$.

A full-text calculus query has the conventional semantics of first-order logic. The form of the quantification in the last bullet guarantees that the query expression in the full-text calculus can be evaluated using only the positions and tokens in the context node, without having to look at other positions. This notion is similar to the notion of safety for the relational calculus.

We now provide some examples of full-text calculus queries. The following query returns the context nodes that contain the tokens 'test' and 'usability'.

$\left\{\right.$ node $\mid$ SearchContext $($ node $) \wedge \exists \operatorname{pos}_{1}\left(\right.$ hasPos $\left(\right.$ node $\left._{\text {pos }}\right) \wedge$ hasToken $\left(\right.$ pos $_{1},{ }^{\prime}$ test $\left.^{\prime}\right) \wedge$ 
$\exists \operatorname{pos}_{2}\left(\right.$ hasPos $\left(\right.$ node, $\left.\operatorname{pos}_{2}\right) \wedge$ hasToken $\left(\right.$ pos $_{2},{ }^{\prime}$ usability') $\left.\left.)\right)\right\}$

In the subsequent examples, we only show the query expression since the rest of the query is the same. The following query returns the context nodes that contain the token 'test' and the token 'usability' with at most 5 intervening tokens.

$\exists \operatorname{pos}_{1}\left(\right.$ hasPos $\left(\right.$ node, $\left.\operatorname{pos}_{1}\right) \wedge$ hasToken $\left(\right.$ pos $_{1}{ }^{\prime}{ }^{\prime}$ test $\left.^{\prime}\right) \wedge \exists \operatorname{pos}_{2}\left(\right.$ hasPos $\left(\right.$ node, pos $\left._{2}\right) \wedge$

hasToken $\left(\right.$ pos $_{2},{ }^{\prime}$ usability $) \wedge$ distance $\left(\right.$ pos $_{1}$, pos $\left.\left.\left._{2}, 5\right)\right)\right)$

The following query returns the context nodes that contain two occurrences of the token 'test' and do not contain the token 'usability'.

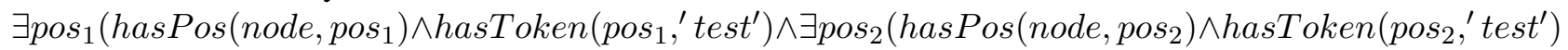

$\wedge$ diffpos $\left(\operatorname{pos}_{1}, \operatorname{pos}_{2}\right) \wedge \forall \operatorname{pos}_{3}\left(\right.$ hasPos $\left(\right.$ node $\left._{10 s_{3}}\right) \Rightarrow \neg$ hasToken $\left(\operatorname{pos}_{3},{ }^{\prime}\right.$ usability $\left.\left.\left.)\right)\right)\right)$

\subsection{Full-Text Algebra}

We now define our full-text relations and algebra operators. The underlying data model for our algebra is a full-text relation of the form $\mathrm{R}\left[\mathrm{CNode}\right.$, att $\mathrm{t}_{1}, \ldots$, at $\left.\mathrm{t}_{\mathrm{m}}\right]$ where the domain of CNode is $\mathcal{N}$ (context nodes), and the domain of att $t_{i}$ is $\mathcal{P}$ (positions). $R$ satisfies the following properties.

- $\mathrm{R}$ has always at least the attribute CNode. This captures the context node for full-text search. The remaining attributes in $R$ capture the essence of full-text search, which is to manipulate positions.

- Each tuple $t$ in a full-text relation should satisfy the condition that for all the positions pos in $t, p o s \in$ Positions(t.CNode). The intuition is that a full-text search query can only manipulate positions within a single context node.

A full-text algebra expression is based on the following full-text relations that characterize the search context nodes, their positions, and the tokens at these positions.

- SearchContext(CNode): This relation contains a tuple (node) for each node $\in \mathcal{N}$.

- HasPos(CNode, att ${ }_{1}$ ): This relation contains a tuple for each (node,pos) pair that satisfies: node $\in$ $\mathcal{N} \wedge$ pos $\in$ Positions (node). Intuitively, this relation relates context nodes to their positions.

- $\mathrm{R}_{\text {token }}\left(\mathrm{CNode}, \mathrm{att}_{1}\right)$ : This is a family of relations, one for each token $\in \mathcal{T} . R_{\text {token }}$ contains a tuple for each (node,pos) pair that satisfies: node $\in \mathcal{N} \wedge$ pos $\in$ Positions (node) $\wedge$ token $=$ Token (pos). Intuitively, $R_{\text {token }}$ contains positions that contain token, and is similar to an inverted list in IR.

We note that while each $R_{\text {token }}$ relation is finite, there number of such relations will be infinite if $\mathcal{T}$ is infinite. However, this does not lead to a problem in defining the algebra because each algebra expression is finite, and can only refer to a finite set of such relations. Also, physically instantiating the potentially infinite set of $R_{\text {token }}$ relations is not a problem because only a finite sub-set of these relations will be non-empty (because the search context is finite), so only this finite set of relations will have to be explicitly stored. This is in fact what happens in current implementations of inverted lists.

In addition, as in the calculus, we have a set of position-based predicates Preds. 


\subsubsection{Full-Text Algebra Operators and Queries}

The full-text algebra operators are similar to the relational operators, but with two important differences. First, full-text algebra operators only operate on full-text relations (as defined above), and not on arbitrary relations, due to the nature of full-text search. Second, full-text algebra operators implicitly enforce that each operation only manipulates positions within a single node, and not across nodes. These two properties ensure that the full-text algebra is equivalent to the full-text calculus in characterizing full-text search. A full-text algebra expression is defined recursively as follows.

- SearchContext is an algebra expression that returns the tuples in the full-text relation SearchContext.

- HasPos is an algebra expression that returns the tuples in the full-text relation HasPos.

- $R_{\text {token }}$ is an algebra expression that returns the tuples in the $R_{\text {token }}$ relation, where token $\in \mathcal{T}$.

- If $\operatorname{Expr}_{1}$ is an algebra expression, $\pi_{\mathrm{CNode}, \mathrm{att}_{1}, \ldots, \mathrm{att}_{\mathrm{i}}}\left(\operatorname{Expr}_{1}\right)$ is an algebra expression. If $\operatorname{Expr}_{1}$ evaluates to the full-text relation $R_{1}$, the full-text relation corresponding to the new expression is: $\pi_{\mathrm{CNode}_{\text {,att }}, \ldots, \text { att }_{\mathrm{i}}}\left(\mathrm{R}_{1}\right)$, where $\pi$ is the traditional relational projection operator. The attribute names of the result full-text relation are renamed to have consecutive at $t_{i}$ 's. Note that $\pi$ always has to include CNode in the full-text algebra - this enforces the property that full-text search is always scoped within a single context node.

- If Expr $r_{1}$ and $E x p r_{2}$ are algebra expressions, then $\left(E x p r_{1} \bowtie E x p r_{2}\right)$ is an algebra expression, If $\operatorname{Expr}_{1}$ and $\operatorname{Expr}_{2}$ evaluate to $R_{1}$ and $R_{2}$ repectively, then the full-text relation corresponding to

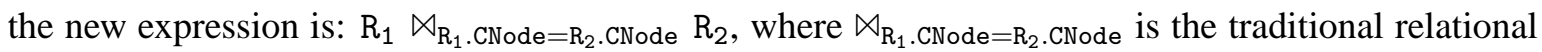
equi-join operation on the CNode attribute. The duplicate CNode attribute is projected out in the result full-text relation, and the position attributes are renamed to be consecutive att $t_{i}$ 's. Note again how the full-text algebra does not allow operations across nodes because the only predicate that is permitted in the join is equality between the attributes CNode of the two relations.

- If $\operatorname{Expr}_{1}$ is an algebra expression, then $\sigma_{\text {pred }\left(\mathrm{att}_{1}, \ldots, \text { att } \mathrm{m}, \mathrm{c}_{1}, \ldots, \mathrm{c}_{\mathrm{q}}\right)}\left(\operatorname{Expr}_{1}\right)$ is an algebra expression, where pred $\in$ Preds. If Expr $r_{1}$ evaluates to $R_{1}$, the full-text relation corresponding to the new expression is: $\sigma_{\text {pred }}\left(\mathrm{att}_{1}, \ldots, \mathrm{att}_{\mathrm{m}}, \mathrm{c}_{1}, \ldots, \mathrm{c}_{\mathrm{q}}\right)\left(\mathrm{R}_{1}\right)$, where $\sigma$ is the traditional relational selection operator.

- If Expr $r_{1}$ and $E x p r_{2}$ are algebra expression, then $\left(\operatorname{Expr}_{1}-\operatorname{Expr}_{2}\right), \operatorname{Expr}_{1} \cup E x p r_{2}$, and Expr $\cap$ $E x p r_{2}$ are algebra expressions. These,$- \cup$ and $\cap$ operators have the same semantics as in traditional relational algebra.

A full-text algebra query is a full-text algebra expression that produces a full-text relation with a single attribute (this attribute has to be CNode by definition). The set of nodes in the result full-text relation defines the result of a full-text algebra query.

We now provide some examples of full-text algebra queries that correspond to the calculus example in Section 2.2.1. The following query returns the context nodes that contain the token 'test' and 'usability': $\pi_{\text {CNode }}\left(R_{\text {test }} \bowtie R_{\text {usability }}\right)$

The following query returns the context nodes that contain the token 'test' and the token 'usability' within a distance of 5: $\pi_{\text {CNode }}\left(\sigma_{\text {distance }\left(p_{1}, p_{2}, 5\right)}\left(\mathrm{R}_{\text {test }} \bowtie \mathrm{R}_{\text {usability }}\right)\right)$

The following query returns the context nodes that contain two occurrences of the token 'test' and do not contain the token 'usability': $\pi_{\mathrm{CNode}}\left(\left(\sigma_{\text {diffpos }\left(\text { att }_{1}, a t t_{2}\right)}\left(\mathrm{R}_{\text {test }} \bowtie \mathrm{R}_{\text {test }}\right)\right) \bowtie\right.$ (SearchContext $\left.\pi_{\text {CNode }}\left(\mathrm{R}_{\text {usability }}\right)\right)$ ) 


\subsection{Equivalence of Calculus and Algebra and Its Applications}

Theorem 1 Given a set of position-based predicates Preds, the full-text calculus and the full-text algebra are equivalent in expressive power.

The proof is in Appendix A, and is similar to the equivalence proof for the relational calculus and algebra.

The equivalence of the full-text calculus and algebra suggests a notion of completeness for full-text search languages. This provides a formal basis for comparing the expressive power of different query languages, as we shall do in the next section. To the best of our knowledge, this is the first attempt to formalize the expressive power of full-text search languages, either for flat documents or for XML documents. Developing a full-text algebra in terms of relations also provides a foundation for tightly integrating, optimizing and evaluating structured (relational or XML) queries with full-text search.

The full-text algebra also enables us to rank query results by leveraging existing work on the probabalistic relational model developed in the context of IR $[19,38]$. Specifically, the probabilistic relational model includes a probability attribute for each tuple that specifies its relevance to the result relation. A tuple with a high probability is very relevant to the result relation, while a tuple with low probability is not. In addition, the model defines how these probabilities are propagated through traditional relational operators. In our context, we simply need to add a new probability attribute to our full-text relations. We can then rely on these techniques to propagate this attribute through the algebra operators, and produce ranked results.

\section{Scoring}

Scoring (or ranking) is an important aspect of full-text search. However, there is no standard agreed-upon method for scoring full-text search results. In fact, developing and evaluating different scoring methods is still an active area of research $[14,18,21,20,27,33,19,38]$. Thus, rather than hard-code a specific scoring method into our framework, we describe a general scoring framework based on the FTC and the FTA, and show how some of the existing scoring methods can be incorporated into this framework. Specifically, we now show how TF-IDF [28] and PRA [19, 38] scoring methods can be incorporated. We only describe how scoring can be done in the context of the FTA; the extension to the FTC is similar.

\subsection{TF-IDF Based Scoring}

TF-IDF is one of the most common IR scoring methods [28].

Our scoring framework is based on two extensions to our model: (1) per-tuple scoring information and (2) scoring transformations. Per-tuple scoring information associates a score with each tuple in a full-text relation, similar to [19]. However, unlike [19], the scoring information need not be only a real number (or probability); it can be any arbitrary type that can be extended for the needs of the scoring method. Scoring transformations extend the semantics of FTA operators to transform the scores of the input full-text relations. For example, a selection operator can scale the scores based in the selection predicate (such as distance) and so on.

We now show how TF-IDF scoring can be captured using our scoring framework. We use the following widely-accepted TF and IDF formulae for a node $n$ and a token $t: t f(n, t)=$ occurs $(n, t) /$ unique_tokens $(n)$ and $i d f(t)=\ln \left(1+d b_{-}\right.$size $\left./ d f(t)\right)$, where occurs $(n, t)$ is the number of occurrences of $t$ in $n$, unique_tokens $(n)$ is the number of unique tokens in $n$, db_size is the number of nodes in the database, and $d f(t)$ is the number of nodes containing the token $t$. The TD-IDF scores are aggregated using the cosine similarity: 
$\operatorname{score}(n)=\Sigma_{t \in q} w(t) * t f(n, t) * i d f(t) /\left(\|n\|_{2} *\|q\|_{2}\right)$, where $q$ denotes the bag of search tokens in the query, $w(t)$ denotes the weight of the search token $t$ and $\|\cdot\|_{2}$ is the $L_{2}$ measure.

To model TF-IDF, we associate a numeric score with each tuple. Intuitively, the score contains the TFIDF measure for all the positions in the tuple. Initially, the $R_{t}$ relations contain the static scores: the $i d f(t)$ for the token $t$ at that position divided by the product of the normalization factors unique_tokens $*\|n\|_{2}$. This is the $L_{2}$ normalized TF-IDF measure for each position containing the token $t$. Thus, if we sum all the scores in $R_{t}$, we get exactly the $L_{2}$-normalized TF-IDF measure of $t$ with regards to $n$. It is also important to note that all of the scoring information in $R_{t}$ can be precomputed.

To capture TF-IDF score of search tokens, the above tuple score can be scaled by weight $(t) /$ (unique_search_tokens $*\|q\|_{2}$ ), where unique_search_tokens is the number of unique search tokens in the query $q$. This scale factor is query-dependent and cannot be precomputed. Thus, we can consider that the persistent index structures contain the $i d f(t) /\left(\right.$ unique_tokens $\left.*\|n\|_{2}\right)$ score. When the $R_{t}$ relation is processed, the precomputed score is multiplied by $i d f(t) /$ unique_search_tokens $*\|q\|_{2}$ to obtain the final score for a tuple $t$ :

$$
\text { t.score }=i d f(t)^{2} /\left(\text { unique_tokens } * \text { unique_search_tokens } *\|n\|_{2} *\|q\|_{2}\right)
$$

We now describe the scoring transformations for each FTA operator.

- Given two expressions $\operatorname{Expr}_{1}$ and $\operatorname{Expr}_{2}$ that evaluate to the full-text relations $\mathrm{R}_{1}$ and $\mathrm{R}_{2}$, a tuple $t_{1}$ in $R_{1}$, a tuple $t_{2}$ in $R_{2}$ and $t_{3}$ in $\left(\operatorname{Expr}_{1} \bowtie E x p r_{2}\right)$ where $t_{3}$ is the result of joining $t_{1}$ and $t_{2}$, i.e., $t_{1} . C N$ ode $=t_{2}$.CNode, the score formula associated with join is:

$$
t_{3} . \text { score }=t_{1} . \text { score } /\left|R_{2}\right|+t_{2} /\left|R_{1}\right|
$$

Above, $|\cdot|$ denotes the cardinality of the relation. We need to scale down the $t_{1}$.score and $t_{2}$.score because their relevance decreases due to the increased number of tuples (solutions) in the resulting relation. Informally, one can think of this as "the first law of thermodynamics" for conservation of energy: the join conserves the total score (energy) of the input relations because it neither adds nor removes solutions (tuples).

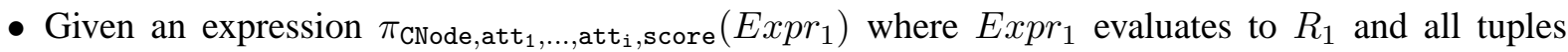
$t_{1} \ldots t_{n}$ in $R_{1}$ that project out onto the same output tuple $t_{3}$ (i.e., they share thesame values for the projected attributes), the score formula associated with projection is:

$$
t_{3} . \text { score }=\Sigma_{i=1, . ., n} t_{i} . \text { score }
$$

Projection also obeys the above score-conservation: the new relation should have the same total score as the original one.

- Given $\sigma_{\text {pred }\left(a t t_{1}, \ldots, a t t_{n}, c_{1}, \ldots, c_{m}\right)}\left(\operatorname{Expr}_{1}\right)$ where $\operatorname{Expr}_{1}$ is an algebra expression whose corresponding full-text relation is $R_{1}$. Let $R_{2}$ is the resulting relation. If $t_{2}$ is a tuple in $R_{2}$ such that $t_{1}=t_{2}$, then:

$$
t_{2} . \text { score }=t_{1} . \text { score }
$$


- Given an expression $\neg E x p r_{1}$ where $E x p r_{1}$ evaluates to $R_{1}$ and $t$ is a tuple in $R_{1}$, the score formula associated with the negation is: t.score $=1-t$.score (tie negation to difference in the definition of the algebra).

- Given $\left(\operatorname{Expr}_{1} \cup E x p r_{2}\right)$ where $\operatorname{Expr}_{1}$ and $E x p r_{2}$ are algebra expressions whose corresponding fulltext relations are $R_{1}$ and $R_{2}$ and $t_{1}$ is a tuple in $R_{1}$ and $t_{2}$ is a tuple in $R_{2}$ and $t_{3}$ is the result of the union of $t 1$ and $t_{2}$, the score formula associated with the union is:

$$
t_{3} . \text { score }=t_{1} . \text { score }+t_{2} \text {.score }
$$

We assume that if $t_{i}$.score $=0$ if $\nexists t_{i} \in R_{i} t_{i}=t_{3}$ for $i=1$, 2 ; i.e., missing tuples are assumed to have score 0 .

- Given $\left(\operatorname{Expr}_{1}-\operatorname{Expr}_{2}\right)$ where Expr $r_{1}$ and Expr 2 are algebra expressions whose corresponding fulltext relations are $R_{1}$ and $R_{2}$. Let $R_{3}$ is the resulting relation. If $t_{3}$ is a tuple in $R_{3}$ such that $t_{1}=t_{3}$, then:

$$
t_{3} . \text { score }=t_{1} . \text { score }
$$

- Similarly, given $\left(E x p r_{1} \cap E x p r_{2}\right)$ where $E_{x p r_{1}}$ and $E x p r_{2}$ are algebra expressions whose corresponding full-text relations are $R_{1}\left(C N o d e, a t t_{1}, \ldots, a t t_{n}\right)$ and $R_{2}\left(C N o d e, a t t_{1}, \ldots, a t t_{n}\right)$. Let $R_{3}$ is the resulting relation. Let $t_{1}$ is a tuple in $R_{1}$ and $t_{2}$ is a tuple in $R_{2}$ such that $t_{1}$.CNode $=$ $t_{2} . C N$ ode, $t_{1} . a t t_{1}=t_{2} . a t t_{1}, \ldots, t_{1} . a t t_{n}=t_{2} . a t t_{n}$, and $t_{3} \in R_{3}$ be the resulting tuple, then:

$$
t_{3} . \text { score }=\operatorname{Min}\left(t_{1} . \text { score }, t_{2} . \text { score }\right)
$$

The following theorem holds.

Theorem 2 The TF-IDF propagation of scores preserves the traditional semantics of TF-IDF for conjunctive and disjunctive queries.

Proof sketch. We consider restricted FTC expressions of the form $\{$ node $\mid$ hasPos(node) $\wedge$ QueryExpr (node)\} where QueryExpr(node) can be one of the following

- hasPos $($ node, $p) \wedge$ hasToken $(p, t)$ for some $p \in \mathcal{P}, t \in \mathcal{T}$

- $\left(\right.$ QueryExpr ${ }_{1}($ node $\left.)\right) \wedge\left(\right.$ Query Expr $_{2}($ node $\left.)\right)$ for some restricted FTC expressions QueryExpr ${ }_{1}($ node $)$ and QueryExpr ${ }_{1}$ (node)

- (QueryExpr $\left.\operatorname{Exode}_{1}\right) \mathrm{V}\left(\right.$ Query Expr $_{2}($ node $\left.)\right)$ for some restricted FTC expressions QueryExpr ${ }_{1}($ node $)$ and QueryExpr ${ }_{1}($ node $)$ 
We assume that all search tokens are distinct. This can be achieved by considering the search token position in the query to be part of the search token. Notice that this does not influence the TF-IDF score of query results. Let two search tokens $t_{1}$ and $t_{2}$ have the same TF measure $t f$ and IDF measure $i d f$. Let the weight of the first one be $w_{1}$ and the weight of the second one be $w_{2}$. Then their combined TF-IDF score is $\left(w_{1} * t f * i d f+w_{2} * t f * i d f\right) /\left(\|n\|_{2} *\|q\|_{2}\right)=\left(w_{1}+w_{2}\right) * t f * u d f /\left(\|n\|_{2} *\|q\|_{2}\right)$, i.e. it is the same as a token with weight $w_{1}+w_{2}$.

We use structural induction on the restricted FTC expression $E$. We will prove the following invariant. Let $E_{1}$ is a subexpression of $E$. Let $A E x p r_{1}$ be its corresponding FTA expression. Then, for every attribute att $t_{i}$ in the resulting relation $R_{1}$ of $A \operatorname{Expr}_{1}$ and its corresponding search token $q_{i}$, the following holds $\forall u \in \pi_{C N o d e, a t t_{i}}\left(A E x p r_{1}\right)$ u.score $=\operatorname{score}\left(u . C N o d e, q_{i}\right)$. Here, $\operatorname{score}\left(n, q_{i}\right)=w\left(q_{i}\right) * t f\left(n, q_{i}\right) *$ $i d f\left(q_{i}\right) /\left(\|n\|_{2} *\|q\|_{2}\right)$ is the score of the search context node $n \in \mathbb{N}$ with respect to the token $q_{i}$.

- Let $E=$ hasPos $($ node, $p) \wedge$ hasToken $(p, t)$ for some $p \in \mathcal{P}, t \in \mathcal{T}$, i.e. we are searching for the token $t$. The corresponding FTA expression is $\pi_{C N \text { ode }}\left(\mathrm{R}_{t}\right)$. The score of every $n \in \mathbb{N}$ is

$$
\begin{aligned}
\operatorname{score}(n) & =\sum_{u \in \mathrm{R}_{t}} \text { u.score }=\sum_{u \in \mathrm{R}_{t}} \frac{i d f(t)^{2}}{\text { unique_tokens } * \text { unique_search_tokens } *\|n\|_{2} *\|q\|_{2}} \\
& =\frac{\text { occurs } * i d f(t) * i d f(t)}{\text { unique_tokens } * \text { unique_search_tokens } *\|n\|_{2} *\|q\|_{2}} \\
& =\frac{w(t) * t f(n, t) * i d f(t)}{\|n\|_{2} *\|q\|_{2}}
\end{aligned}
$$

This is exactly the TF-IDF score with respect to the search token $t$.

- Let $E=\left(\right.$ QueryExpr $r_{1}($ node $\left.)\right) \wedge\left(\right.$ QueryExpr $_{2}($ node $\left.)\right)$. Let QueryExpr ${ }_{1}($ node $)$ and QueryExpr $2($ node $)$ have corresponding FTA expressions $A E x p r_{1}$ and $A E x p r_{2}$ respectively. Let $R_{1}$ and $R_{2}$ be the results of the evaluation of $A E_{x p r_{1}}$ and $A E_{\text {xpr }}$. Remember that the search tokens (i.e. postition attributes in the resulting full-text relations) are distinct. As in the proof of Theorem 1, the FTC expression $E$ evaluates to the relation $R\left(C N o d e, a t t_{1}, \ldots, a t t_{n}\right)$ that is the result of $A \operatorname{Expr}_{1} \bowtie A E x p r_{2}$.

Let $a t t_{i}$ is a position attribute of $R$. Without loss of generality, att $t_{i}$ also belongs to the relation $R_{1}$. Using the induction hypothesis, we get that $\forall u \in \pi_{C N o d e, \text { att }_{i}}\left(A E \operatorname{Expr}_{1}\right) u$.score $=\operatorname{score}\left(u . C N o d e, q_{i}\right)$.

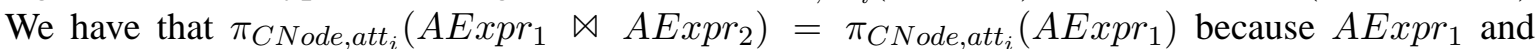
$A E_{x p r_{2}}$ evaluate to relations that have no position attributes in common. Furthermore, for every tuple $u \in R_{1}$, there exist exactly $\left|R_{2}\right|$ tuples $v_{1}, \ldots, v_{\left|R_{2}\right|}$, each with score $u$.score $/\left|R_{2}\right|$, such that $u . C N o d e=v_{j} . C N o d e \wedge u . a t t_{i}=v_{j} . a t t_{i}$ for $j=1, \ldots,\left|R_{2}\right|$. Consequently, $\sum_{j=1}^{\left|R_{2}\right|} v_{j}$. score $=$ u.score $=$ score $\left(u . C N\right.$ ode,$\left.q_{i}\right)$.

Let $v \in \pi_{C N o d e, a t t_{i}}\left(A \operatorname{Expr}_{1} \bowtie A \operatorname{Expr}_{2}\right)=\pi_{C N o d e, a t t_{i}}\left(A \operatorname{Expr}_{1}\right)$. Thus, there exist tuples $v_{1}, \ldots, v_{\left|R_{2}\right|}$ such that $v . C N o d e=v_{j} . C N o d e \wedge v \cdot a t t_{i}=v_{j}$. att $_{i}$ for $j=1, \ldots,\left|R_{2}\right|$. Therefore, v.score $=$ $\sum_{j=1}^{\left|R_{2}\right|} v_{j}$.score $=\operatorname{score}\left(u . C N o d e, q_{i}\right)$.

- The case $E=\left(\right.$ QueryExpr $_{1}($ node $\left.)\right) \vee($ QueryExpr 2 (node $\left.)\right)$ is similar to the previous one.

QED 
Further, this scoring method is more powerful than traditional TF-IDF because it can be generalized to arbitrarily complex queries (not just simple conjunctive and disjunctive queries) by defining appropriate scoring transformations for the other operators. For instance, we can define a scoring transformation for distance selection predicates thereby extending the scope of TF-IDF scoring.

\subsection{Probability Based Scoring}

One of the popular scoring methods employed by the IR community is using probability-based measures to indicate the relevance of a context node to a full-text search condition. The formal underpinnings for this work is specified by the probabilistic relational model $[19,38]$. Specifically, this model includes a probability attribute for each tuple that specifies its score (relevance) to the result relation. A tuple with a high probability score is very relevant to the result relation, while a tuple with low probability score is not. In addition, the model defines how these probabilities are propagated through traditional relational operators.

It is easy to incorporate the above probability-based scoring in the FTA; we simply need to add a new probability attribute to the full-text relations. This new attribute will represent the probability (score) of each tuple in the full-text relation. Since all FTA operations are specified in terms of relational algebra operations, we can directly use the techniques in the probabilistic relational model to propagate the scores for arbitrarily complex FTA expressions.

The probabilistic relational algebra is the most prominent scoring method in full-text search [19]. This algebra operates on tuples with a score attribute. The score of a tuple represents the probability associated with that tuple. A score formula is associated with each operator with transforms its input tuples scores into output tuples scores. We adapt the relational probabilistic model to our algebra. Every full-text relation $\mathrm{R}_{\text {token }}$, where token $\in \mathcal{T}$, is augmented with a score attribute. Conceptually, the score of a tuple in $R_{\text {token }}$ represents the probability that that tuple contains token. Hence, the value of score should be a float between 0 and 1 . This value can be computed using a variety of techniques, including TF and IDF [31]. For example, if TF-IDF is used, then the score of each tuple could be defined as IDF/NF, where NF is the normalizing factor used in computing the TF-IDF score (using the formula TF*IDF/NF). We associate a score formula with each operator in our algebra. Each formula guarantees that output tuples will have a score value between 0 and 1 . In the following, we assume that every full-text relation $R_{i}$ has a score attribute.

- Given an expression $\pi_{\mathrm{CNode}_{2}, \mathrm{att}_{1}, \ldots, \text { att }}, \mathrm{score}\left(\operatorname{Expr}_{1}\right)$ where $\operatorname{Expr}_{1}$ evaluates to $\mathrm{R}_{1}$ and all tuples $t_{1} \ldots t_{n}$ in $\mathrm{R}_{1}$ that project out onto the same output tuple $t_{3}$ (i.e., they share thesame values for the projected attributes), the score formula associated with projection is:

$t_{3}$. score $=1-\left(1-t_{1}\right.$. score $) \times\left(1-t_{2}\right.$. score $) \times \ldots \times\left(1-t_{m}\right.$. score $)$

This formula aggregates the scores of input tuples whose value is between 0 and 1 into a single score whose value is between 0 and 1 .

- Given two expressions $\operatorname{Expr}_{1}$ and $\operatorname{Expr}_{2}$ that evaluate to the full-text relations $\mathrm{R}_{1}$ and $\mathrm{R}_{2}$, a tuple $t_{1}$ in $\mathrm{R}_{1}$, a tuple $t_{2}$ in $\mathrm{R}_{2}$ and $t_{3}$ in $\left(\operatorname{Expr}_{1} \bowtie E x p r_{2}\right)$ where $t_{3}$ is the result of joining $t_{1}$ and $t_{2}$, i.e., $t_{1}$.CNode $=t_{2}$.CNode, the score formula associated with join is:

$t_{3} . s c o r e=t_{1}$.score $\times t_{2}$. score Note that the join preserves the fact that the score of tuples has to be a value between 0 and 1 .

- Given an expression $\sigma_{\text {pred }}\left(\mathrm{att}_{1}, \ldots, \mathrm{att}_{\mathrm{m}}, \mathrm{c}_{1}, \ldots, \mathrm{c}_{\mathrm{q}}\right)\left(\operatorname{Expr}_{1}\right)$ where $\operatorname{Expr}_{1}$ evaluates to $\mathrm{R}_{1}$, the score formula associated with a predicate depends on the predicate pred. Therefore, given a tuple $t$ in $\mathrm{R}_{1}$, its score is defined as follows: 
t.score $=$ t.score $\times f$ where $f$ is a function associated with the predicate and evaluates to a value between 0 and 1 . For example, the function associated with the predicate $\operatorname{distance}\left(p_{1}, p_{2}\right.$, dist $)$ is: $f=1-\left|t \cdot p_{1}-t \cdot p_{2}\right| /$ dist.

- Given an expression $\neg E x p r_{1}$ where $\operatorname{Expr}_{1}$ evaluates to $\mathrm{R}_{1}$ and $t$ is a tuple in $\mathrm{R}_{1}$, the score formula associated with the negation is: t.score $=1-t$.score (tie negation to difference in the definition of the algebra).

- Given $\left(\operatorname{Expr}_{1} \cup \operatorname{Expr}_{2}\right)$ where $\operatorname{Expr}_{1}$ and $\operatorname{Expr}_{2}$ are algebra expressions whose corresponding fulltext relations are $\mathrm{R}_{1}$ and $\mathrm{R}_{2}$ and $t_{1}$ is a tuple in $\mathrm{R}_{1}$ and $t_{2}$ is a tuple in $\mathrm{R}_{2}$ and $t_{3}$ is the result of the union of $t 1$ and $t_{2}$, the score formula associated with the union is: $t_{3}$.score $=1-\left(1-t_{1}\right.$.score $) \times$ $\left(1-t_{2}\right.$. score $)$

- Given two expressions $\operatorname{Expr}_{1}$ and $\operatorname{Expr}_{2}$ that evaluate to the full-text relations $\mathrm{R}_{1}$ and $\mathrm{R}_{2}$, a tuple $t_{1}$ in $\mathrm{R}_{1}$, a tuple $t_{2}$ in $\mathrm{R}_{2}$ and $t_{3}$ in $\left(E x p r_{1} \cap E x p r_{2}\right)$ where $t_{3}$ is the result of joining $t_{1}$ and $t_{2}$, i.e., $t_{1}$.CNode $=t_{2}$.CNode, the score formula associated with join is: $t_{3}$.score $=t_{1}$. score $\times t_{2}$.score Intuitively, the intersection can be interpreted as a join on all attributes.

- The score for the case Expr $r_{1}-E x p r_{2}$ can be derived using Expr $r_{1}-E_{x p r_{2}}=E x p r_{1} \cap \neg E x p r_{2}$.

\section{Completeness of Full-text Search Languages}

In this section, we show the incompleteness of existing full-text languages with respect to the algebra and calculus. We then define a complete full-text language based on the full-text calculus that naturally generalizes existing languages.

\subsection{Incompleteness of Boolean Full-Text Search Languages}

Boolean full-text search languages are commonly used in IR, and have also been proposed for XML fulltext search [18, 33]. A typical syntax for such languages, which we shall call BOOL, is given below. The simplest query is a search token, which can either be a string literal (such as 'test') or the keyword ANY, which matches any token in a node. In addition, the query can be composed with Boolean operators.

Query := Token | NOT Query | Query AND Query | Query OR Query

Token := StringLiteral $\mid$ ANY

We can recursively define the semantics of BOOL in terms of our calculus. If the query is a StringLiteral 'token', it is equivalent to the calculus query expression $\exists p\left(\operatorname{hasPos}(n, p) \wedge\right.$ hasToken $\left.\left(p,{ }^{\prime} \operatorname{token}^{\prime}\right)\right)$. If the query is ANY, it is equivalent to the expression $\exists p(h a s P o s(n, p))$. If the query is of the form NOT Query, it is equivalent to $\neg$ Expr, where Expr is the calculus expression for Query. If the query is of the form Query1 AND Query2, it is equivalent to Expr $1 \wedge \operatorname{Expr} 2$, where Expr 1 and Expr 2 are calculus expressions for Query 1 and Query 2 respectively. OR is defined similarly. As an example, the query ' test' AND NOT ' usability' is equivalent to the calculus query expression: $\exists p_{1}\left(\operatorname{has} \operatorname{Pos}\left(n, p_{1}\right) \wedge\right.$ hasToken $\left(p_{1},{ }^{\prime}\right.$ test $\left.\left.{ }^{\prime}\right)\right) \wedge \neg\left(\exists p_{2}\right.$ hasPos $\left(n, p_{2}\right) \wedge$ hasToken $\left(p_{2},{ }^{\prime}\right.$ usability $\left.\left.{ }^{\prime}\right)\right)$.

Obviously, BOOL cannot express position-based predicates. However, we now show that even if we disallow such predicates in the calculus (i.e., Preds $=\phi$ ), BOOL is still incomplete if $\mathcal{T}$ is infinite.

Theorem 3 If $\mathcal{T}$ is infinite, there exists a full-text query that can be expressed in the full-text calculus with Preds $=\phi$, but which cannot be expressed by BOOL. 
Proof Sketch: We shall show that no query in BOOL can express the following calculus query:

$\exists p\left(\right.$ hasPos $(n, p) \wedge \neg$ hasToken $\left.\left(p, t_{1}\right)\right)$ (i.e., find context nodes that contain at least one token that is not $\left.t_{1}\right)$, where $t_{1} \in \mathcal{T}$. The proof is by contradiction. Assume that there exists a query $\mathcal{Q}$ in BOOL that can express the calculus query. Let $\mathcal{T}_{\mathcal{Q}}$ be the set of tokens that appear in $\mathcal{Q}$. We construct two context nodes $C N_{1}$ and $C N_{2}$. $C N_{1}$ contains only the token $t_{1}$. $C N_{2}$ contains the token $t_{1}$ and one other token $t_{2} \in \mathcal{T}-\left(\mathcal{T}_{\mathcal{Q}} \cup\left\{t_{1}\right\}\right)$ (such a token $t_{2}$ always exists because $\mathcal{T}$ is infinite and $\mathcal{Q}$ is finite). By the construction, we can see that $C N_{1}$ does not satisfy the calculus query, while $C N_{2}$ does. We will now show that $\mathcal{Q}$ either returns both $C N_{1}$ or $C N_{2}$ or neither of them; since this contradicts our assumption, this will prove the theorem.

Let $C_{Q}$ be the calculus expression equivalent to $\mathcal{Q}$. We show by induction on the structure of $C_{Q}$ that every sub-expression of $C_{Q}$ (and hence $C_{Q}$ ) returns the same Boolean value for $C N_{1}$ and $C N_{2}$. If the sub-expression is of the form $\exists p(\operatorname{hasPos}(n, p) \wedge \operatorname{hasToken}(p$, token $))$, it returns true for both $C N_{1}$ and $C N_{2}$ if token $=t_{1}$, and false if token $\neq t_{1}$ (by construction of $C N_{1}$ and $C N_{2}$ - recall that token appears in $\mathcal{Q})$. If the sub-expression is of the form $\exists p(\operatorname{hasPos}(n, p))$, it returns true for both $C N_{1}$ and $C N_{2}$. If the sub-expression is of the form $\neg E x p r$, then it returns the same Boolean value for both $C N_{1}$ and $C N_{2}$ because Expr returns the same Boolean value (by induction). A similar argument can also be made for the $\wedge$ and $\vee$ Boolean operators.

If we limit $\mathcal{T}$ to be finite, however, we can prove that BOOL is complete with Preds $=\phi$.

Theorem 4 If $\mathcal{T}$ is finite, every query that can be expressed in the full-text calculus with Preds $=\phi$ can be expressed in BOOL.

The proof is presented in Appendix A. The main intuition is that, if $\mathcal{T}$ is finite, we can express queries such as: $\exists p\left(\right.$ hasPos $(n, p) \wedge \neg$ hasToken $\left.\left(p, t_{1}\right)\right)$ in BOOL by explicitly listing all the tokens that are not $t_{1}$. Although BOOL is complete under this assumption, it is not always practical because even for simple queries such as the one above, we need to explicitly list all possible tokens other than $t_{1}$ in the query.

\subsection{Incompleteness of Existing Predicate-Based Full-Text Search Languages}

We now consider full-text languages that have position-based predicates in addition to Boolean operators [3, 7]. A typical syntax for such a language, which we shall call DIST, is given below.

Query := Token | NOT Query $\mid$ Query AND Query $\mid$ Query OR Query $\mid$ dist(Token,Token,Integer)

Token $:=$ StringLiteral $\mid$ ANY

The semantics of DIST is the same as BOOL, except for the addition of dist (Token, Token, Integer). This construct is the equivalent of the distance predicate introduced in the calculus (Section 2.2), and specifies that the number of intervening tokens should be less than the specified integer. More formally, the semantics of $\operatorname{dist}\left(t_{1}, t_{2}, d\right)$ for some tokens $t_{1}$ and $t_{2}$ and some integer $d$ is given by the calculus expression: $\exists p_{1}\left(\operatorname{hasPos}\left(n, p_{1}\right) \wedge\right.$ hasToken $\left.\left(p_{1}, t_{1}\right) \wedge \exists p_{2}\left(\operatorname{hasPos}\left(n, p_{2}\right) \wedge \operatorname{hasToken}\left(p_{2}, t_{2}\right) \wedge \operatorname{distance}\left(p_{1}, p_{2}, d\right)\right)\right)$. If $t_{1}$ or $t_{2}$ is ANY instead of a string literal, then the corresponding hasToken predicate is omitted in the semantics. We now show that DIST is incomplete with respect to the calculus so long as $\mathcal{T}$ is not trivially small. We can also prove similar incompleteness results for other position-based predicates.

Theorem 5 If $|\mathcal{T}| \geq 2$, there exists a full-text query that can be expressed in the full-text calculus with Preds $=\left\{\right.$ distance $\left.\left(p_{1}, p_{2}, d\right)\right\}$, but which cannot be expressed by DIST.

Proof Sketch: We shall show that no query in DIST can express the following calculus query: $\exists p_{1}\left(\operatorname{hasPos}\left(n, p_{1}\right) \wedge \exists p_{2}\left(\operatorname{hasPos}\left(n, p_{2}\right) \wedge\right.\right.$ hasToken $\left.\left.\left(p_{1}, t_{1}\right) \wedge \operatorname{hasToken}\left(p_{2}, t_{2}\right) \wedge \neg \operatorname{distance}\left(p_{1}, p_{2}, 0\right)\right)\right)$, where $t_{1} \in \mathcal{T}, t_{2} \in \mathcal{T}$ and $t_{1} \neq t_{2}$ (i.e., find context nodes where the tokens $t_{1}$ and $t_{2}$ do not appear next 
to each other at least once). The proof is by contradiction. Assume that there exists a query $\mathcal{Q}$ in DIST that can express the calculus query. We now construct two context nodes $C N_{1}$ and $C N_{2}$ as follows. $C N_{1}$ contains the tokens $t_{1}$ followed by $t_{2}$ followed by $t_{1}$. $C N_{2}$ contains the tokens $t_{1}$ followed by $t_{2}$ followed by $t_{1}$ followed by $t_{2}$. By the construction, we can see that $C N_{1}$ does not satisfy the calculus query, while $C N_{2}$ does. Using induction on the structure of $\mathcal{Q}$ similar to the proof of Theorem 3, we can show that $\mathcal{Q}$ either returns both $C N_{1}$ or $C N_{2}$ or neither of them. This is a contradiction.

\subsection{A Complete Full-Text Query Language}

We now present a new language COMP based on the full-text calculus that is complete even in the presence of arbitrary position-based predicates. COMP shares the same syntax as BOOL for simple Boolean queries, but naturally generalizes BOOL with position variables to achieve completeness. Thus, simple queries retain the same conventional syntax, while new constructs are only required for more complex queries.

Query := Token | NOT Query | Query AND Query | Query OR Query | SOME Var Query | EVERY Var Query | Preds

Token := StringLiteral | ANY | Var HAS StringLiteral | Var HAS ANY

Preds := distance(Var,Var,Integer) $\mid$ diffpos(Var,Var) $\mid$...

The main additions to BOOL are the HAS construct in Token, and the SOME, EVERY and Preds constructs in Query (the semantics of the other constructs remain unchanged from BOOL). The HAS construct allows us to explicitly bind position variables (Var) to positions where tokens occur. The semantics for ' $v^{2} r_{1}$ HAS tok' in terms of the calculus, where tok is a StringLiteral is: hasToken $\left(v r_{1}, t_{o k}\right)$. The semantics for 'var ${ }_{1}$ HAS ANY' is: hasPos $\left(n, v_{a r}\right)$. While the HAS construct allows us to explicitly bind position variables to token positions, the SOME and EVERY constructs allows us to quantify over these positions. The semantics of 'SOME $\operatorname{var}_{1}$ Query' is $\exists v a r_{1}\left(h a s P o s\left(n, v^{2} r_{1}\right) \wedge E x p r\right)$, where Expr is the calculus expression semantics for Query. The semantics of 'EVERY $\operatorname{var}_{1}$ Query' is $\forall \operatorname{var}_{1}\left(h_{a s P o s}\left(n, \operatorname{var}_{1}\right) \Rightarrow \operatorname{Expr}\right)$, where Expr is the calculus expression semantics for Query. Finally, the Preds construct allows for the definition of arbitrary position-based predicates. The semantics of a predicate ' $\operatorname{pred}\left(\operatorname{var}_{1}, \ldots, v a r_{p}, c_{1}, \ldots c_{q}\right)$ ', is simply: $\operatorname{pred}\left(\operatorname{var}_{1}, \ldots, \operatorname{var}_{p}, c_{1}, \ldots, c_{q}\right)$.

As an illustration of the power of COMP, the following two queries express the calculus queries used to prove the incompleteness of BOOL and DIST in Theorems 3 and 5, respectively.

SOME $p_{1}$ (NOT $p_{1}$ HAS $t_{1}$ )

SOME $p_{1}$ SOME $p_{2}\left(p_{1}\right.$ HAS $t_{1}$ AND $p_{2}$ HAS $t_{2}$ AND NOT distance $\left.\left(p_{1}, p_{2}, 0\right)\right)$

We can prove that COMP is complete (the proof is in the appendix).

Theorem 6 Every query that can be expressed in the full-text calculus using predicates Preds can be expressed by COMP using Preds.

\section{Query Complexity and Evaluation Algorithms}

While one important aspect of a full-text language is expressibility (discussed in the previous section), another important aspect is query complexity, i.e., the efficiency of evaluating a query in a full-text language. In this section, we study the query complexity of different full-text languages and develop efficient query evaluation algorithms. Due to space constraints, we will only sketch the algorithms to evaluate NPRED queries.

Like other formal languages, full-text languages have a tradeoff between expressibility and query complexity: the more expressive the language, the greater its query complexity. We formalize this notion by developing a complexity hierarchy of full-text languages based on the inverted list [28] model for query 
evaluation commonly used in the IR community (see Section 5.1.2). At the top of our complexity hierarchy is COMP, which is the most expressive but which also has the greatest query complexity. At the bottom of the hierarchy is BOOL, which is the least expressive but also has the lowest query complexity. We also identify two new classes of languages between these two extremes: PPRED, which stands for a subset of COMP restricted to "Positive" PREDicates, and NPRED, which stands for a subset of COMP restricted to "Negative" PREDicates (we shall formally define positive and negative predicates in Sections 5.5 and 5.6). PPRED includes most common full-text predicates, such as distance and samepara, but is more powerful than existing full-text languages such as DIST. NPRED is a superset of PPRED and includes the negations of most common full-text predicates.

An interesting result of our study is that the query evaluation complexity of PPRED is linear in the size of the query token inverted lists, and quadratic in the size of the query. Specifically, in Section 5.5, we present an algorithm whereby PPRED queries can be evaluated in a single scan over the query term inverted lists. This illustrates a practical application of our formalism: developing full-text languages such as PPRED that are more powerful than existing full-text predicate languages (such as DIST), but which can still be evaluated efficiently in a single scan over inverted lists. Similarly, we also show in Section 5.6, that the query evaluation complexity of NPRED is linear in the size of the query inverted lists but, in some case, exponential in the size of the query - this additional complexity is the price paid for negation.

We note that our focus in this section is on establishing query complexity upper bounds for the various full-text languages by developing concrete, efficient, and practical query evaluation algorithms (especially for PPRED and NPRED). Exploring query complexity lower bounds is beyond the scope of this paper, and is part of future work. We now start by describing our complexity model.

\subsection{Complexity Model}

Our study of the complexity of full-text search languages is similar in spirit to the vast body of work on the complexity of database query languages (e.g., $[8,9,23,36])$. However, there are two main reasons why the complexity results for database query languages do not directly apply to our setting.

First, our focus is specifically on full-text search using the inverted list model for data organization (which is the commonly used model in the IR community). Thus, our complexity parameters are expressed in terms of this model, and we establish upper bounds by developing concrete query evaluation algorithms based on this model. In contrast, most database query languages work with arbitrary relations (not just full-text relations and inverted lists); while this leads to more general results, these results do not isolate the complexity of full-text primitives in the context of the inverted list model.

Second, most database query language complexity studies treat expression complexity (i.e., the complexity of evaluating a query as a function of the size of the query, assuming the database is the same) [9, 36] and data complexity $[23,36]$ (i.e., the complexity of evaluating a query as a function of the size of the data, assuming that the query is the same) separately. In contrast, we are interested in combined complexity (defined, but not explored in [36]), whereby we want to determine the complexity of evaluating a query as a function of both the query size and the data size in order to study their relative impact on query performance.

\subsubsection{Query Model}

We characterize a COMP query Q by the following parameters. Since the other full-text languages that we consider are subsets of COMP, these parameters apply to these languages as well.

- $\operatorname{toks}_{Q}$ : The number of tokens in $\mathrm{Q}$, including string literals and the universal token ANY. 


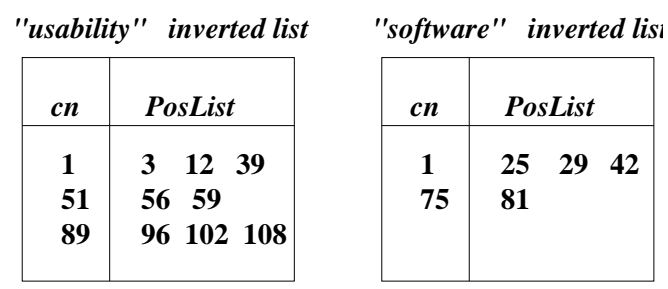

Figure 2: Inverted Lists Examples

- $\operatorname{pred} s_{Q}$ : The number of predicates in $\mathrm{Q}$.

- ops $_{Q}$ : The number of operations in Q, where an operation can be NOT, AND, OR, SOME, or EVERY.

The above parameters characterize the total size of a COMP query since they capture all the primitives that can appear in a query.

\subsubsection{Data Model}

As mentioned earlier, we use the inverted list model [28] for representing context nodes. For each token tok that appears in at least one context node in $\mathcal{N}$, there is an associated inverted list $I L_{t o k} . I L_{t o k}$ contains a list of one or more entries. Each entry is a pair: (cn,PosList), where $c n$ is the id of a context node that contains tok, and PosList is the list of positions in $\mathrm{cn}$ that contain tok. The positions in PosList are ordered based on their order of occurrence in $\mathrm{cn}$, and the entries in $I L_{t o k}$ are ordered based on the ids of the context nodes. Intuitively, $I L_{t o k}$ corresponds to the physical representation of the full-text relation $R_{t o k}$ in the FTA. Figure 2 shows example inverted lists for the usability and software tokens, where the document in Figure 1 is one of the context nodes and has id 1.

In addition to the inverted lists, there is also a list, $I L_{A N Y}$, which contains one entry for each context node in $\mathcal{N}$. Each entry is the pair: (cn, PosList), where $c n$ is the id of a context node, and PosList is the list of positions that occur in $\mathrm{cn}$. Again, the positions in PosList are ordered based on their order of occurrence in $c n$, and the entries in $I L_{A N Y}$ are ordered based on the ids of the context nodes. Intuitively, $I L_{A N Y}$ corresponds to the physical representation of the ANY full-text relation in the FTA.

One important restriction on inverted lists is that they can only be accessed sequentially (some IR implementations allow restricted random accesses, but we do not consider these extensions here). Specifically, the only way to access an inverted list $I L_{t o k}$ (similarly, for $I L_{A N Y}$ ) is to open a cursor. Each cursor sequentially scans $I L_{t o k}$ and supports the following two operations.

- nextEntry(): The first nextEntry() call moves the cursor to the first entry $e$ in $I L_{t o k}$, and returns the id of the context node in $e$. Each subsequent call advances the cursor to the next entry in $I L_{t o k}$ and returns the corresponding context node id. When all entries have been scanned, nextEntry() returns NULL. The entry pointed to by the cursor at any time is called the current entry.

- getPositions(): This call returns the list of all positions (PosList) in a given entry int he inverted list.

We assume that each invocation of the above operations is executed in $O(1)$ (i.e., constant) time.

Finally, to quantify the size of the inverted lists, we use the following parameters. We use $\mathcal{T}$ to denote the set of all tokens that appear in the context nodes $\mathcal{N}$. 
- cnodes: $|\mathcal{N}|$ (the number of context nodes).

- pos_per_cnode: $\max _{(c n, P o s L i s t) \in I L_{A N Y}}(\mid$ PosList $\mid)$ (the maximum number of positions in a context node).

- entries_per_token: $\max _{t o k \in \mathcal{T}}\left(\left|\left\{e \mid e \in I L_{t o k}\right\}\right|\right)$ (the maximum number of entries in a token inverted list).

- pos_per_entry: $\max _{t o k \in \mathcal{T}} \max _{(\mathrm{cn}, \text { PosList }) \in I L_{t o k}}(\mid$ PosList $\mid)$ (the maximum number of positions in an entry in a token inverted list).

We note that the above parameters are conservative in the sense that they use the maximum values for the number of positions per context node, etc.; we do this to keep the model simple. However, as we shall soon see, these conservative parameters are still sufficient to clearly separate the query evaluation complexity of the full-text languages that we consider.

\subsection{Summary of Complexity Results}

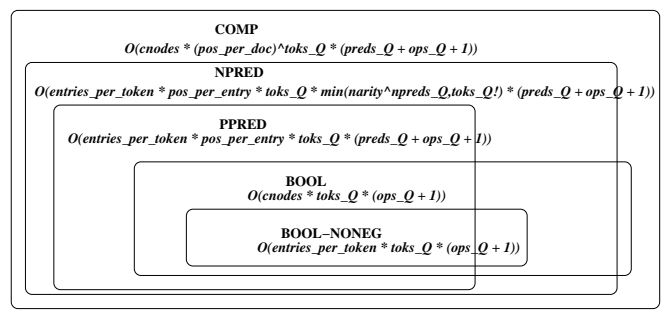

Figure 3: Complexity Hierarchy

Figure 3 summarizes our complexity results; we present the details in the subsequent sections. We represent each language by a bounding box and depict the query complexity of that language (expressed in terms of our complexity parameters) within the bounding box; note that these are upper bounds on the query complexity. If the bounding box of a language $\mathrm{A}$ encloses the bounding box of another language $\mathrm{B}$, then all queries in B can be expressed in A. If the bounding boxes of two languages A and B intersect, but no one bounding box contained in the other, then there are some queries that can be expressed in A but not in B, and vice versa.

As shown, the main results are:

- The query complexity of COMP is polynomial in the size of the inverted lists and exponential in the size of the query.

- The query complexity of BOOL without negation (BOOL-NONEG) is linear in the size of the query token inverted lists, and linear in the size of the query.

- The query complexity of BOOL (with negation) is linear in the size of the ANY list, and linear in the size of the query.

- The query complexity of PPRED is linear in the size of the query token inverted lists, and linear in the size of the query. 
- The query complexity of NPRED is linear in the size of the query token inverted lists, and possibly exponential in the size of the query.

The above complexity results demonstrate the potential for performance savings using PPRED and NPRED: they reduce the query complexity from polynomial in the size of the data (for COMP) to linear in the size of the data.

We now discuss the complexity results and query evaluation algorithms in more detail. Due to space constraints, we only briefly discuss BOOL and COMP, and focus mainly on developing efficient query evaluation algorithms for PPRED. We will only sketch the implementation of NPRED due to space limitations.

\subsection{BOOL: Evaluation and Complexity}

As mentioned in Section 4.1, BOOL is commonly used in IR systems. We first consider a subset of BOOL called BOOL-NONEG, which does not have ANY and does not allow NOT as the first operator. It has the following grammar (note that NOT can only appear along with an AND).

Query := Token | Query AND NOT Query | Query AND Query | Query OR Query

Token := StringLiteral

A common way to evaluate queries in the above language is to merge [28] the inverted lists for the query tokens. For example, consider the query ('software' AND 'users' AND NOT 'testing') OR ' usability'. The query can be evaluated by merging $I L_{\text {software }}$ and $I L_{\text {users }}$ (for the first AND) to determine the context node ids that contain both tokens. This result can then be merged with $I L_{\text {testing }}$ to determine the context node ids that do not contain 'testing' (for NOT). Finally, this result can be merged with $I L_{\text {usability }}$ to determine the union of the context node ids (for OR). Since the inverted list entries are sorted by the context node ids, each merge can be done in a single scan over the query token inverted lists. Since the total size of the relevant parts of the query token inverted lists is entries_per_token $\times$ toks $s_{Q}$ (since BOOL-NONEG ignores positions), and each inverted list entries are scanned at most once for each operator, the query evaluation complexity of BOOL-NONEG is: $O\left(\right.$ entries_per_token $\times$ toks_Q $\times\left(o p s_{Q}+1\right)$.

In contrast to BOOL-NONEG, BOOL allows ANY and NOT to appear anywhere in the query (Section 4.1). Since ANY and NOT require access to $I L_{A N Y}$ (to find all positions in a context node), and $I L_{A N Y}$ has cnodes entries, the query complexity of BOOL is: $O\left(\right.$ cnodes $\times$ toks $_{-} Q \times\left(\right.$ ops $\left.\left._{Q}+1\right)\right)$.

A scoring formula is associated with each Boolean operator in BOOL and BOOL-NONEG as is defined in [19]. Initially, a score is associted with each entry in the inverted lists and are modified by each Boolean operator in the query plan.

\subsection{COMP: Evaluation and Complexity}

As discussed in Section 4.3, COMP has a one-to-one mapping to the FTC. Since the FTC is a Quantified Boolean Formula (QBF), it is LOGSPACE-complete for data complexity (complexity in the size of the database) and PSPACE-complete for expression complexity (complexity in the size of the query) [36]. It is an open question as to whether LOGSPACE is a strict subset of PTIME (polynomial time), and whether PSPACE is a strict subset of EXPTIME (exponential time). Thus, for all practical purposes given our current knowledge, we can only devise a query evaluation algorithm that is polynomial in the size of the data and exponential in the size of the query. We now outline one such algorithm.

The basic idea is to translate the FTC expression corresponding to a COMP query into an equivalent FTA expression (using the equivalence of FTC and FTA given in Section 2.4). The FTA expression can then be evaluated using regular relational operators. As an illustration, consider the following COMP query: SOME $p_{1} \operatorname{SOME} p_{2}$ ( $p_{1}$ HAS 'usability' AND $p_{2}$ HAS 'software' AND 


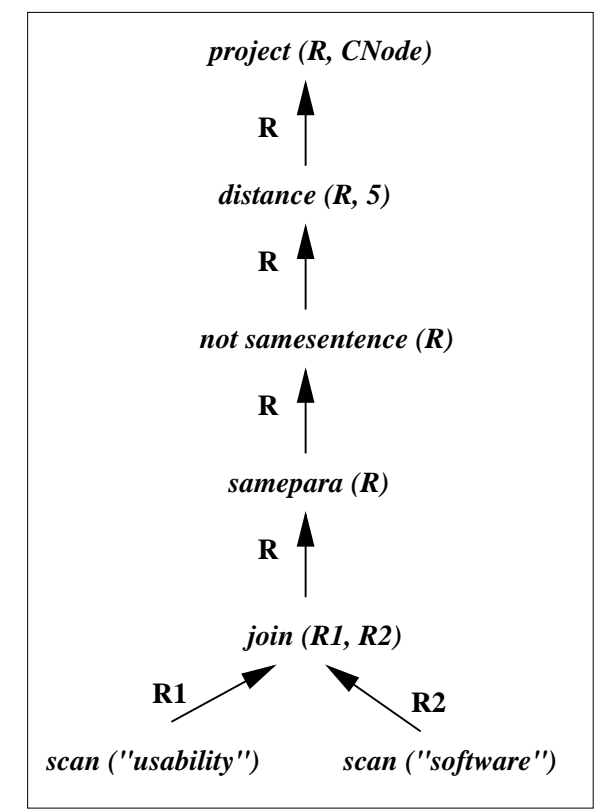

Figure 4: Query Plan Example

samepara $\left(p_{1}, p_{2}\right)$ AND $\neg$ samesentence $\left(p_{1}, p_{2}\right)$ AND distance $\left(p_{1}, p_{2}, 5\right)$ ) (return context nodes that contain the words "usability" and "software" in the same paragraph, in that order, within at most 5 words of each other). The resulting FTA expression tree is shown in Figure 4.

The complexity of evaluating a COMP query is thus bounded by the complexity of the FTA operators. Since all FTA operators except the join operator have complexity linear in the size of their input, we focus on the join operator. If the join operator takes in two inputs $I$ and $J$, and $I$ has $p$ tuples for context node $c n$, and $J$ has $q$ tuples for context node $c n$, then the result has $p \cdot q$ tuples for context node $c n$ (since the full-text join operator always performs an equi-join on the context nodes). Thus, the worst case complexity of a join is a cartesian product of the number of tuples per context node. Since there can be at most toks $_{Q}$ joins in a COMP query, and the query can access the $I L_{A N Y}$ relation in general (with size cnodes $\times$ pos_per_cnode), the complexity of a COMP is:

$O\left(\right.$ cnodes $\times(\text { pos_per_cnode })^{\text {toks }_{\mathbf{Q}}} \times\left(\right.$ preds $_{\mathbf{Q}}+$ ops $\left.\left._{\mathbf{Q}}+1\right)\right)$

Scoring in COMP is handled by each operator in the query plan as defined in Section 3. This also applies to PPRED and NPPRED.

\subsection{PPRED: Evaluation and Complexity}

PPRED is a subset of COMP that restricts the use of negation and only allows "positive" predicates (which will shall formalize soon), which actually include most common predicates used in the IR community. The surprising aspect of PPRED is that, by placing these restrictions, it can guarantee that queries can be run in linear time over the size of the query inverted lists, instead of in polynomial time; for large, practical data sets, this translates to a huge gain in performance. The grammar for P PRED is given below, where Query* refers to a Query with no free variables.

Query := Token | Query AND NOT Query* | Query AND Query | Query OR Query | SOME Var Query | EVERY Var Query $\mid$ Preds

Token $:=$ StringLiteral | Var HAS StringLiteral 
Preds $:=$ distance(Var,Var,Integer) $\mid$ ordered(Var,Var) $\mid \ldots$

Like BOOL-NONEG, PPRED only allows negations to appear in the context of an AND and cannot explicitly specific ANY. Both of these restrictions ensure that $I L_{A N Y}$ does not need to be accessed during query processing. Since query operations can be implemented in a linear scan over the query inverted lists in the presence of "positive" predicates, we have the following query complexity for PPRED:

$$
O\left(\text { entries_per_token } \times \text { pos_per_entry } \times \text { toks }_{Q} \times\left(\operatorname{preds}_{\mathbf{Q}}+\mathbf{o p s}_{\mathbf{Q}}+1\right)\right)
$$

We now describe the intuition behind positive predicates and how it enables an efficient linear query evaluation algorithm. We then formalize the properties and algorithms.

\subsubsection{Positive Predicates: Intuition}

Intuitively, positive predicates are those that are true in a contiguous region in the position space, and are false outside of this region. For instance, the distance predicate is true in the region where both positions are within the distance limit, and false outside this region. A more complex example of a positive predicate is ordered, where the region specifies the part of the position space where the positions are in the required order. Other common full-text predicates such as samepara, window, etc. are also positive predicates. Given positive predicates, how can we use their property to devise efficient query evaluation algorithms?

Recall from the complexity discussion in COMP that the main source of complexity stems from the evaluation of the join operation, which computes the cartesian product of the number of tuples per context node. If the query contains only positive predicates, we can avoid computing this cartesian product, while still producing the correct results. The key idea is to skip over continuous regions of positions in the cartesian product by exploiting the property of positive predicates, without missing any answer to a query. This skipping over is done in increasing order of positions, and hence can be done in a linear scan over the inverted lists.

As an illustration, consider the following query: SOME $p_{1}$ SOME $p_{2} \quad\left(p_{1}\right.$ HAS ' usability' AND $p_{2}$ HAS 'software' AND distance $\left(p_{1}, p_{2}, 5\right)$ ) (return context nodes that contain the words "usability" and "software" within at most 5 words of each other). Consider evaluating the query over the inverted lists shown in Figure 2. A naive evaluation plan would be to join $I L_{\text {usability }}$ and $I L_{\text {testing }}$ on the context node, and compute the cartesian product of positions, and then apply the distance predicate. For the context node with id 1, this corresponds to computing 9 pairs of positions ( 3 in each inverted list), and then only selecting the final pair $(39,42)$ that satisfies the distance predicate. However, it is sufficient to determine the answer by only scanning 6 pairs of positions $(3+3$ instead of $3 * 3)$.

Specifically, we start with the smallest pair of positions $(3,25)$ and check whether it satisfies the distance predicate. Since it does not, we move the smallest position to get $(12,25)$. Since this does not satisfy the predicate again, we move the smallest position to get $(39,25)$, and so on until we find the solution $(39,42)$. Note that we only scan each inverted list position exactly once, so the complexity is linear in the size of the inverted lists. The reason we were able to move the smallest position is because the distance predicate is true in a contiguous region, and if the predicate is false for the smallest position in the region, we can infer that it is also false for other positions without having to explicitly enumerate them.

\subsubsection{Positive Predicates: Definition}

We now formally define positive predicates. 
Definition 1 [Positive Predicates] A n-ary position-based predicate pred is said to be a positive predicate iff there exists $n$ functions $f_{i}: \mathcal{P}^{n} \rightarrow \mathcal{P}(1 \leq i \leq n)$ such that:

$$
\begin{gathered}
\forall p_{1}, \ldots, p_{n} \in \mathcal{P}\left(\neg \operatorname{pred}\left(p_{1}, \ldots, p_{n}\right) \Rightarrow\right. \\
\forall i \forall p_{i}^{\prime} \in \mathcal{P} \\
\forall p_{i} \leq p_{i}^{\prime}<f_{i}\left(p_{1}, \ldots, p_{n}\right) \Rightarrow \\
\forall p_{1-1}^{\prime}, \ldots, p_{i+1}^{\prime}, \ldots, p_{n}^{\prime} \in \mathcal{P} \\
p_{1} \leq p_{1}^{\prime}, \ldots, p_{i-1} \leq p_{i-1}^{\prime}, \\
p_{i+1} \leq p_{i+1}^{\prime}, \ldots, p_{n} \leq p_{n}^{\prime} \Rightarrow \neg \operatorname{pred}\left(p_{1}^{\prime}, \ldots, p_{n}^{\prime}\right) \\
\wedge \\
\quad \exists j f_{j}\left(p_{1}, \ldots, p_{n}\right)>p_{j}
\end{gathered}
$$

Intuitively, the property states that for every tuple of positions that do not satisfy the predicate (a) there exists a contiguous area, in which all tuples do no satisfy the predicate; this area is specified in terms of the functions $f_{i}\left(p_{1}, \ldots, p_{n}\right)$, which specifies the lower bound of the next possible solution, and (b) at least one $f_{i}\left(p_{1}, \ldots, p_{n}\right)$ has value greater than $p_{i}$; this specifies which position inverted list can be moved forward without compromising correctness.

As mentioned earlier, predicates such as distance, samepara, ordered are positive predicates. For instance, for the 2-ary distance predicate (we only count position parameters in the arity), $f_{1}\left(p_{1}, p_{2}\right)=p_{1}+1$ if $p_{2}>p_{1}$, and $=p_{1}$ otherwise. Similary, $f_{2}\left(p_{1}, p_{2}\right)=p_{2}+1$ if $p_{1}>p_{2}$, and $=p_{2}$ otherwise. samepara and ordered have similar $f_{i}$ functions.

\subsubsection{PPRED Query Evaluation}

We now present the PPRED query evaluation algorithm. Like COMP, we generate a query evaluation tree with We can now describe the evaluation algorithm for P R RED queries. However, since we do not want to materialize the entire output full-text relational corresponding to an operator, each operator exposes a new API for traversing its output full-text relation. This API ensures that successive calls can be evaluated in a linear scan over the inverted list. If the output full-text relation for an operator $o$ is $R$, and has $n$ position columns, then the API for traversing $R$ has the following structure and semantics.

The API maintain the following state: cnode, which tracks the current context node, and $p_{1}, \ldots, p_{n}$, which track the current positions in cnode. It has the following methods:

- advanceCnode(): On the first call, it sets cnode to be the smallest $c$ such that $c \in \pi_{\text {Cnode }}(R)$ (if one exists; else cnode is set to NULL). It also the set values of positions, $p_{1}, \ldots, p_{n}$ such that: (cnode, $\left.p_{1}, \ldots, p_{n}\right) \in R \wedge \forall p_{1}^{\prime}, \ldots, p_{n}^{\prime}\left(\right.$ cnode $\left., p_{1}^{\prime}, \ldots, p_{n}^{\prime}\right) \in R \Rightarrow p_{1}^{\prime} \geq p_{1} \wedge \ldots \wedge p_{n}^{\prime} \geq p_{n}$ (i.e., it sets positions $p_{1}, \ldots p_{n}$ to be the smallest positions that appear in $R$ for that cnode; we will always be able to find such positions due to the property of positive predicates). On subsequent calls, cnode is updated to the next smallest $c \in \pi_{\text {Cnode }}(R)$ (if one exists), and $p_{1}, \ldots, p_{n}$ are updated as before.

- getCnode(): Returns the current value of cnode.

- advancePosition(i,pos): It sets the values of $p_{1}, \ldots, p_{n}$ such that they satisfy: (cnode $\left., p_{1}, \ldots, p_{n}\right) \in$ $R \wedge p_{i} \geq \operatorname{pos} \wedge \forall p_{1}^{\prime}, \ldots, p_{n}^{\prime}\left(\right.$ cnode $\left., p_{1}^{\prime}, \ldots, p_{n}^{\prime}\right) \in R \wedge p_{i}^{\prime} \geq \operatorname{pos} \Rightarrow\left(p_{1}^{\prime} \geq p_{1} \wedge \ldots \wedge p_{n}^{\prime} \geq p_{n}\right)$ (i.e., the smallest values of positions that appear in $R$ and that satisfy the condition $p_{i}>p o s$ ), and returns true. If no such positions exist, then it sets $p_{i}$ s to be NULL and returns false.

- getPosition(i): Returns the current value of $p_{i}$. 


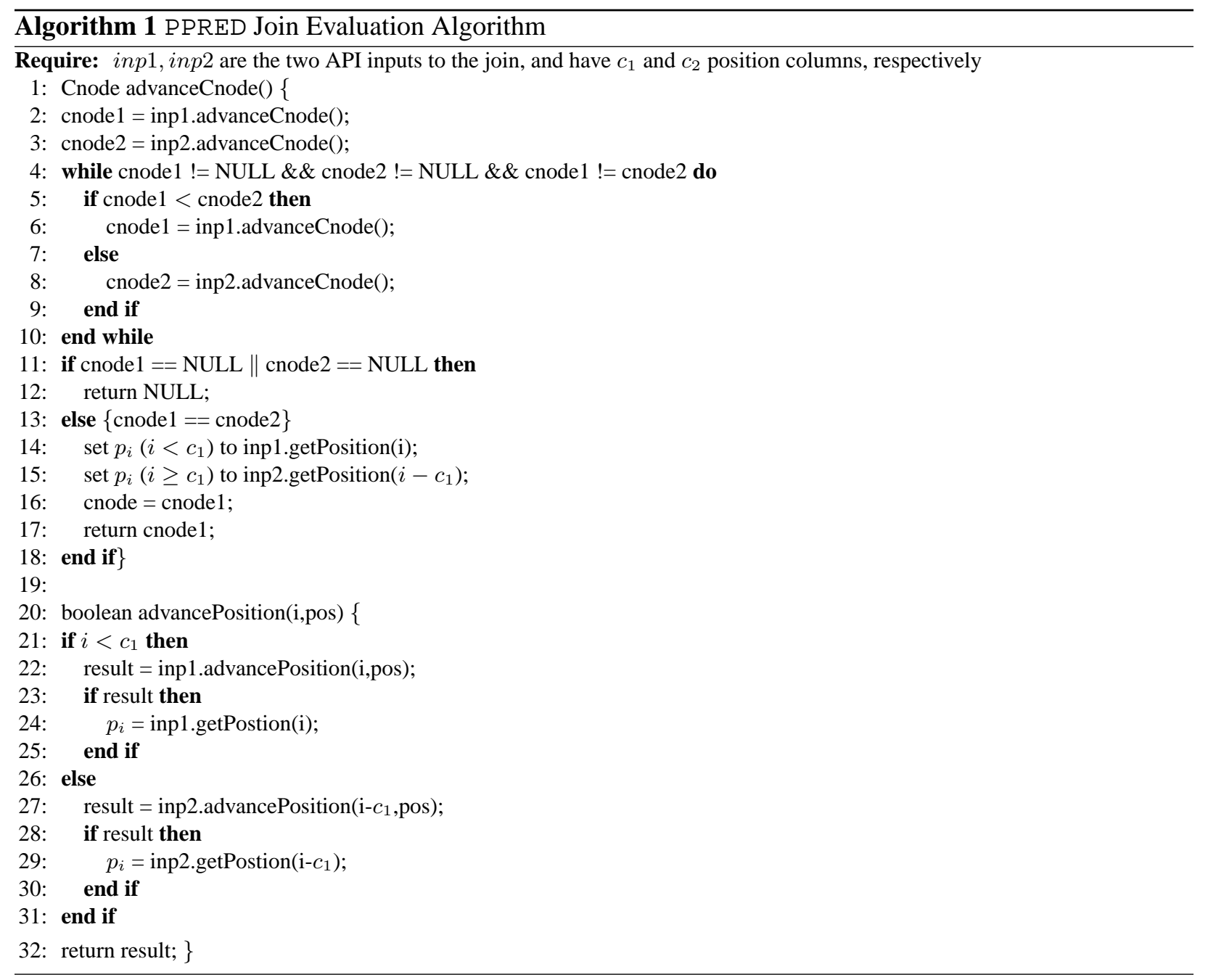

We now show how the different operators can implement the above API in PPRED. The API implementation for the inverted list scan and project operators are straighforward since they directly operate on the inverted list and input operator API, respectively. Thus, we focus on the join operator and the select operator (for evaluating predicates). The implementation for set difference and union is similar to join, and is not discussed here.

Algorithm 1 shows how the API is implemented for the join operator. We only show the implementation of the advanceCnode and advancePos methods since the implementation of the other methods is trivial. The advanceCnode method works as follows. On each invocation, it invokes the advanceCnode method on its inputs until it finds a Cnode that appears in both inputs; this intuitively performs an equijoin on the cnode. It then sets the positions $p_{i}$ to the corresponding positions in the input. The advancePosition ( $i$, pos) method works as follows. It invokes advancePosition on the $i$ 'th position in the corresponding input, and sets the position accordingly. Intuitively, this just moves the position cursor on the corresponding input.

Algorithm 1 shows how the API is implemented for the select operator implementing predicate pred with functions $f_{i}$ (see definition in Section 5.5.2). The advanceCnode method works as follows. On each invocation, it invokes the advancecnode method on its input. It then invokes the helper method 


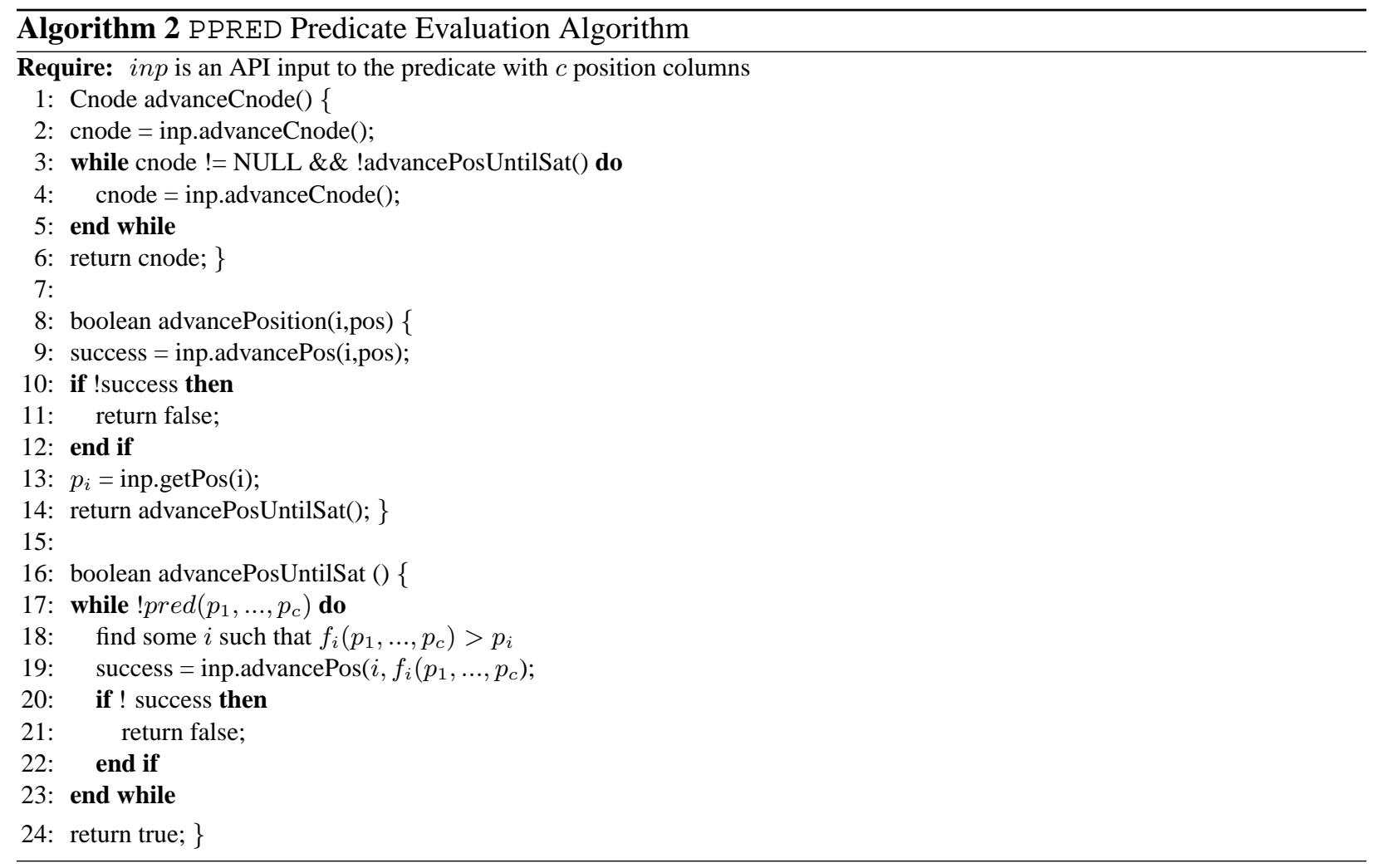

advancePosUntilSat, which returns true iff it is able to advance the positions of the current cnode so that they satisfy the predicate pred. If positions satisfying the predicate for the current cnode are not found, then it advances cnode until one that satisfies the predicate is found, or there are no cnodes left. Essentially, the algorithm loops until a set of positions satisfying the predicate are found.

The advancePosUntilsat method works as follows. It first checks whether the current positions satisfy pred. If not, it uses the $f_{i}$ functions to determine an $i$ whose positions can be advanced, advances that position in the input, and loops back to check whether the new positions satisfy pred. This is done until a set of positions satisfying pred are found, or until no more positions are available. Note that this is the core operation in the select operator: scanning the input positions until a match is found. The properties of positive predicates enable us to do this in a single pass over the input. The implementation of the advancePos method is similar. It first advances the position on its input, and then invokes advancePosUntilsat until a set of positions that satisfy pred are found.

We have proved the correctness of the PPRED query evaluation algorithm, and have also shown that the algorithm has the asymptotic time complexity mentioned in the beginning of this section.

\subsection{NPRED: Evaluation and Complexity}

We now discuss NPRED, its query evaluation and complexity. NPRED is a superset of PPRED, and has the same grammar but handles a larger class of predicates. Specifically, NPRED supports "negative" predicates in addition to the positive predicates supported by P RED. Intuitively, negative predicates are used to model the negations of common IR predicates, such as NOT distance and NOT samepara.

The formal definition of negative predicates is essentially the inverse of the definition of positive predi- 
cates.

Definition 2 [Negative Predicates] A n-ary position-based predicate pred is said to be a negative predicate iff

$$
\begin{aligned}
& \forall p_{1}, \ldots, p_{n} \in \mathcal{P} \neg \operatorname{pred}\left(p_{1}, \ldots, p_{n}\right) \Rightarrow \\
& \quad \exists i_{1}, \ldots, i_{n} p_{i_{1}} \leq \ldots \leq p_{i_{n}} \wedge \forall p_{i_{n}}^{\prime} \in \mathcal{P} p_{i_{1}} \leq p_{i_{n}}^{\prime} \leq p_{i_{n}} \Rightarrow \\
& \forall p_{i_{1}}^{\prime}, \ldots, p_{i_{n-1}}^{\prime} \in \mathcal{P} p_{i_{1}} \leq p_{i_{1}}^{\prime}, \ldots, p_{i_{n-1}}^{\prime} p_{i_{1}}^{\prime} \leq \ldots \leq p_{i_{n-1}}^{\prime} \leq p_{i_{n}}^{\prime} \Rightarrow \neg \operatorname{pred}\left(p_{1}^{\prime}, \ldots, p_{n}^{\prime}\right)
\end{aligned}
$$

Intuitively, the above property says the following. For negative predicates, if a set of positions does not satisfy the predicate, every set of positions between the smallest and the largest one will not satisfy either. Thus, we can satisfy the predicate only by extending the interval between the smallest and the largest position. Compare this to the case of positive predicates, where we the goal was to contract this interval.

The addition of negative predicates to the query language increases the complexity of query evaluation. The intuitive reason is easy to see: consider the evaluation of the query SOME $p_{1} \mathrm{SOME} p_{2} \quad\left(p_{1}\right.$ HAS ' software' AND $p_{2}$ HAS 'usability' AND NOT distance $\left.\left(p_{1}, p_{2}, 5\right)\right)$. Obviously, we cannot use the PPRED evaluation stragegy (Section 5.5.1) of moving the smallest of the two positions $p_{1}$ and $p_{2}$, because the values of $p_{1}$ and $p_{2}$ may always occur close together, and that may not satisfy the NOT distance predicate. In contrast, what we wish to do is fix $p_{1}$ and move only $p_{2}$ until NOT distance is satisfied, or fix $p_{2}$ and move $p_{1}$ until NOT distance is satisfied.

But which of $p_{1}$ or $p_{2}$ do we fix and which one do we move? In general, we have to try both alternatives because other predicates (such as ordered) in the query may be satisfied for one ordering but not the other, and we may not know apriori what that ordering is. Consequently, instead of scanning the inverted lists just once, we have to scan them as many times as the arity of the negative predicate (in this case, 2).

Below we present an algorithm for evaluating NPRED queries. It resolves the undeterminism outlined in the previous paragraph by running $n$ ! threads of the evaluation algorithm, where $n$ is the number of search tokens. Each thread uses an ordering permutation $i_{1}, \ldots, i_{n}$ of $\{1, \ldots, n\}$. The latter specifies an ordering of the cursors over the inverted lists. If $p_{1}, \ldots, p_{n}$ are the current positions, then the invariant is that $p_{i_{1}} \leq \ldots \leq p_{i_{n}}$. Thus, when trying to satisfy a negative predicate, the algorithm moves always the iterator over the inverted list that points to the largest position.

It must be noted that the presented algorithm is not the most efficient. We need orderings only among positions that are used in negative predicates, i.e. we need a partial order among these the positions. On the other hand, the ordering permutation imposes a total order which is needed only if all positions are used in negative predicates. We chose to present this less efficient algorithm because it demonstrates the main points of the query evaluation while keeping the presentation simple. An actual implementation may perform a static analysis of the query and generate only the necessary partial order.

The query evaluation algorithm for NPRED is similar to PPRED with two exceptions: (1) the largest position is moved in NPRED selection operators to try and violate the predicate, and (2) multiple scans of the inverted list are performed, as described above. As in the previous section, we describe the join algorithm and the predicate evaluation algorithm.

Algorithm 3 presents the join algoritm for NPRED. The advanceCnode method is identical to the PPRED case and is omitted. The advancePosition method is also similar to the one used for PPRED but it also ensures that the positions are always in the order specified by the permutation $i_{1}, \ldots, i_{n}$.

Algorithm 4 presents the predicate evaluation algorithm for NPRED differs from Algorithm 2 only in the advancePosUntilsat method. Thus, we present only this method. Unlike advancePosUntilsat for positive predicates, advancePosUntilsat for negative predicates always moves the cursor pointing to the largest position. 


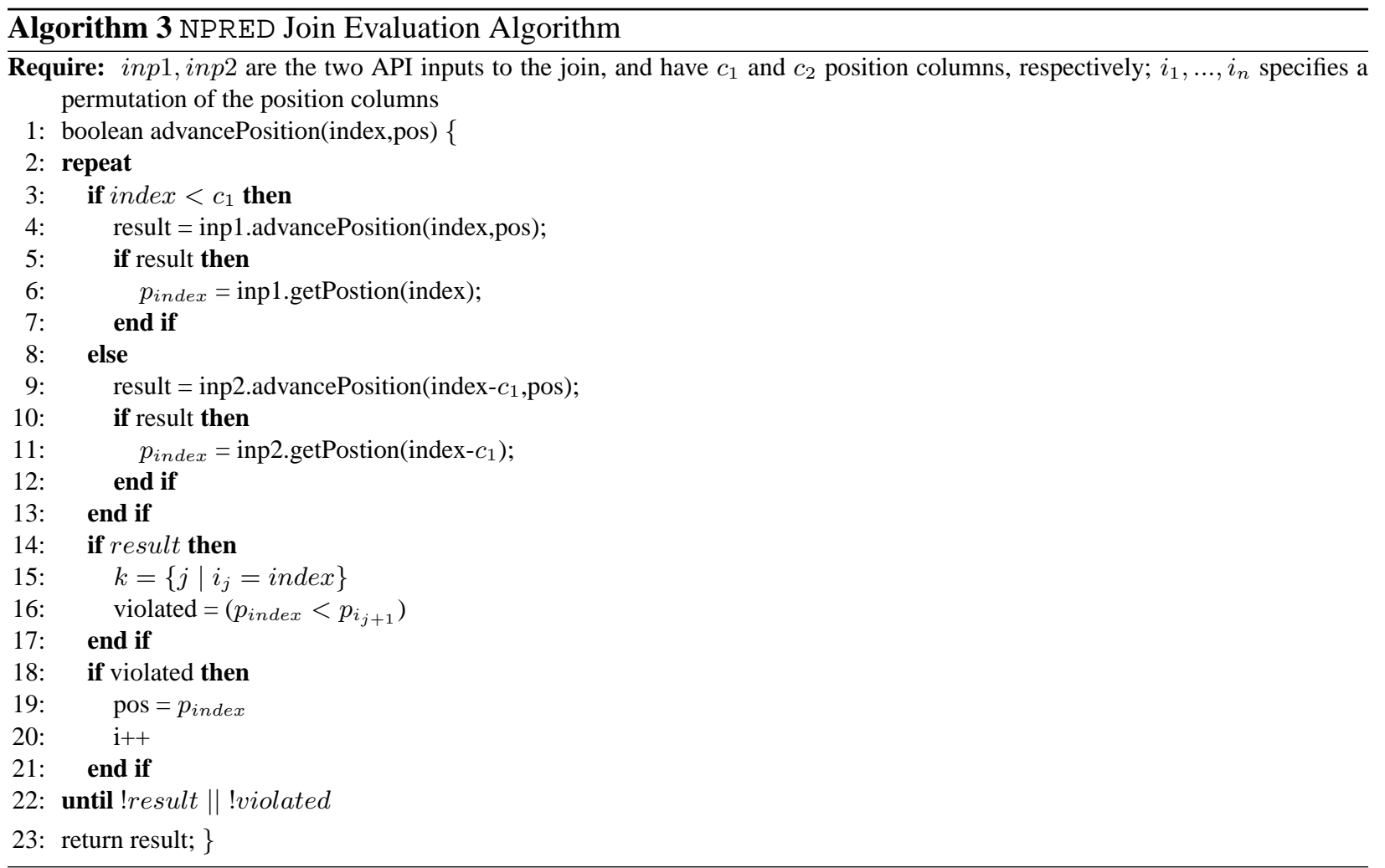

To determine the complexity of the above evaluation algorithm, we remember that we need at most toks $s_{Q}$ ! orderings of positions (one for each ordering of positions in different inverted lists), the total complexity of NPRED is:

$O\left(\right.$ entries_per_tok $\times$ pos_per_entry $\times$ toks $\times \min \left(\right.$ narity $^{\text {npreds }} \mathbf{Q}$, toks $\left._{\mathbf{Q}} !\right) \times\left(\right.$ preds $_{\mathbf{Q}}+$ ops $_{\mathbf{Q}}+$ 1))

\section{Experiments}

We performed experiments on both real and synthetic data sets. Due to lack of space, we report our experiments on real data and only mention similar results on synthetic data. The goals of our experiments are

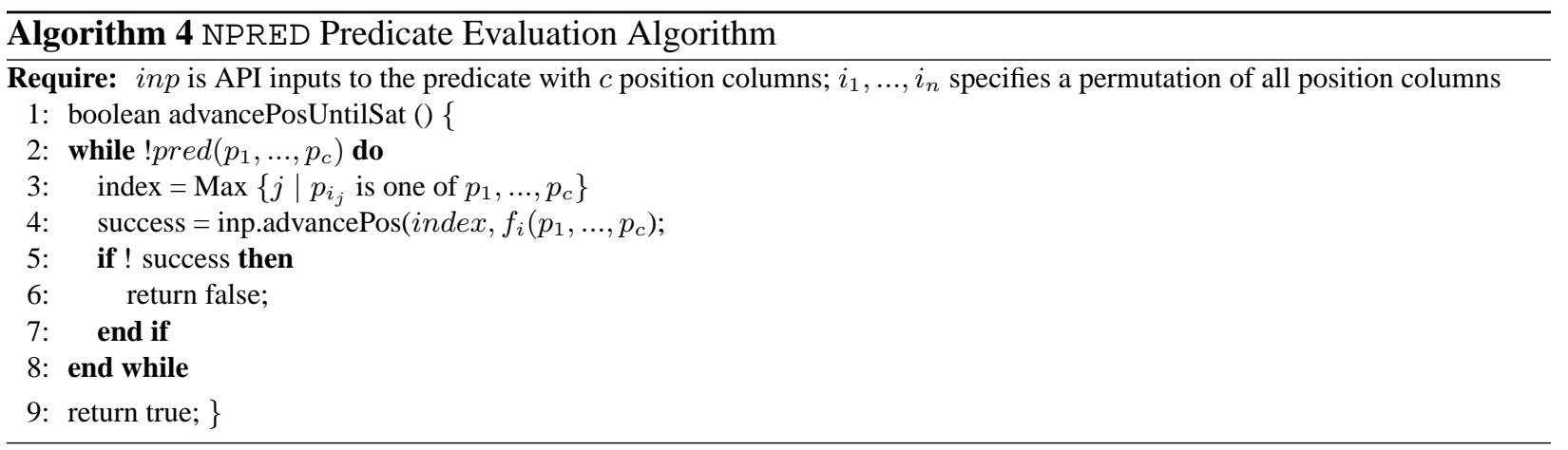


(1) to compare the performance of the evaluation algorithms in Section 5 and study the trade-offs between language expressiveness and complexity and, (2) to study the effect of the query parameters listed below on each algorithm.

- tok $_{\mathrm{Q}}$ : The number of tokens in $\mathrm{Q}$, including string literals and the universal token ANY.

- $\operatorname{pred}_{\mathrm{Q}}$ : The number of predicates in $\mathrm{Q}$.

- op$_{\mathrm{Q}}$ : The number of operations in $\mathrm{Q}$, where an operation can be NOT, AND, OR

\subsection{Summary of Results}

Our results validate the complexity study presented in Section 5 . We show that we can order our algorithms by performance: $\mathrm{BOOL} \preceq \mathrm{PPRED} \preceq$ NPRED $\preceq$ COMP. This was expected given the expressibility of the languages. On the other hand, the interesting fact is that PPRED achieves greater expressibility (the ability to evaluate predicates) than BOOL at a marginally larger cost. On average, PPRED performs better than NPRED for positive predicates due to the fact that PPRED does not need to generate all permutations of the inverted lists. In general, NPRED has noticeably better performance than COMP for both positive and negative predicates. We also observe that in practice, our algorithms perform better than their worst case complexity. In particular, COMP might find a solution early and hence, avoid performing a Cartesian product which explains that sometimes COMP is not much worse than NPRED. Our experiments also show that all of the algorithms perform very similarly when queries do not contain predicates.

\subsection{Experimental Setup}

We implemented the algorithms for BOOL, PPRED, NPRED, and COMP in C++. The evaluation algorithm for BOOL follows the method outlined in the example in Section 5.3. The algorithm for COMP converts the query to an FTA expression and evaluates the latter as in the relational algebra. We ran our experiments on a AMD64 3000+ computer with 1GB RAM and one 200GB SATA drive, running under Linux 2.6.9.

To quantify the size of the scanned inverted lists, we use the following parameters ( $\mathcal{T}$ denotes all tokens that appear in the context nodes $\mathcal{N}$ ).

- cnodes: $|\mathcal{N}|$ (the number of context nodes).

- pos_per_cnode: $\max _{(c n, P o s L i s t) \in I L_{A N Y}}(\mid$ PosList $\mid)$ (the maximum number of positions in a node).

- entries_per_token: $\max _{t o k \in \mathcal{T}}\left(\left|\left\{e \mid e \in I L_{t o k}\right\}\right|\right)$ (the maximum number of entries in a token inverted list).

- pos_per_entry: $\max _{t o k \in \mathcal{T}} \max _{(\mathrm{cn}, \text { PosList }) \in I L_{t o k}}$

$(\mid$ PosList $\mid)$ (the maximum number of positions in an entry in a token inverted list).

We present the experiments for the effects of the $\operatorname{tok}_{\mathbf{Q}}$, $\operatorname{pred}_{\mathbf{Q}}$, cnodes, and pos_per_entry query parameters given above. The experiments on the other parameters supported the conclusions from the summary above and are omitted in the interest of space. To test the influence of each parameter on query evaluation performance, we fixed the other parameters to their default values and varied the values of the studied parameter. In particular, we used: queries with 1 to 5 query tokens (default 3 ) to test tok $\mathrm{k}_{\mathrm{Q}}$; queries with 0 to 4 predicates (default 2) to test pred $_{\mathrm{Q}} ; 2500,6000$ (default), and 10000 context nodes to test cnodes; query tokens with at most 5, 25 (default), and 125 positions per inverted list entry to test pos_per_entry. 


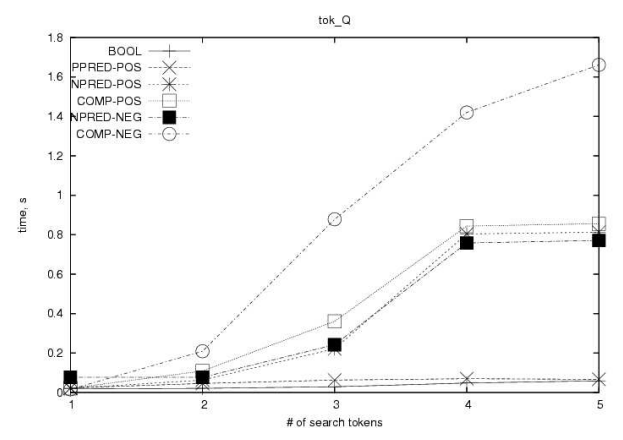

Figure 5: Varying Number of Query Tokens (INEX)

In order to understand the comparative behavior of our algorithms, we plot them all on the same graph. Each algorithm is run with a different query. While the labels BOOL, PPRED, NPRED and COMP represent each algorithm, PPRED-POS, NPRED-POS and COMP-POS (resp., NPRED-NEG and COMP-NEG) report queries with positive predicates only (resp., negative predicate only) for each algorithm. Since the performance of our algorithms is similar when queries have no predicates, we only report BOOL for such queries.

\subsection{Data, Queries and Results}

We used the INEX 2003 XML document collection dataset ${ }^{1}$ which is 500MB large with a little over 12000 documents that contain articles from 17 IEEE journals between 1997 and 2001. Since we are interested in full-text search, we ignored the XML structure and indexed the documents as flat.

Figure 5 shows the performance of our algorithms when varying the number of tokens in the input query and keeping the input data fixed. Figure 6 shows the performance of our algorithms when varying the number of predicates in the input query and keeping the input data fixed. Both experiments show that BOOL and PPRED grow slowly linearly in each of the query size parameters, while COMP and NPRED grow exponentially, the former is faster. Both figures show that PPRED can achieve greater expressibility than BOOL at marginally worse performance.

The big difference in the evaluation time for positive and negative predicates can be explained with the difference in the selectivity of negative predicates: it is higher than the selectivity of the positive predicates. In fact, we used the negation of the positive predicates to generate the negative predicates queries. This explains why the performance of COMP - NEG is bad: large selectivity means it needs to scan many tuples to find a solution. In this case, NPRED is better than COMP for the same queries because it does a more intelligent scan of the inverted lists. It "searches" for the solution, while COMP just blindly enumerates the entire join. The pruning that does NPRED decreases significantly the influence of selectivity.

Although not reported, our experiments on synthetically generated data had similar results when varying the number of tokens and the number of predicates in queries.

Figure 7 shows the performance of our algorithms when varying the number of context nodes. As it can be seen, PPRED and BOOL offer the best scalability: slow linear decrease in performance. The scalability of NPRED is acceptable (the evaluation time increases linearly in the size of the database) while COMP does not scale very well - exponentially. The results for the scalability when we increase the number of positions per inverted list entry (Figure 8) show similar results. This directly influences the size of the join of the inverted

\footnotetext{
${ }^{1}$ http://www.is.informatik.uni-duisburg.de/projects/inex03/
} 


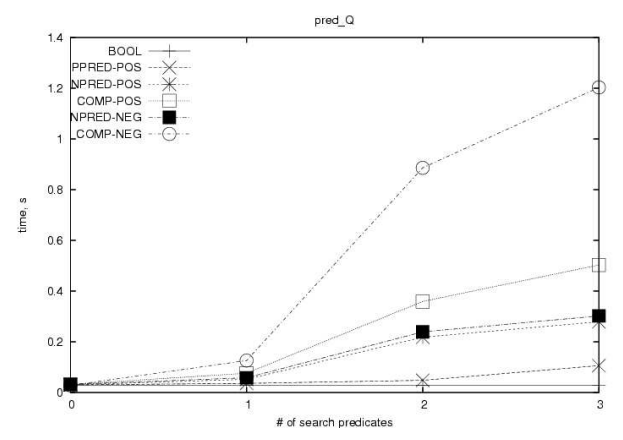

Figure 6: Varying Number of Query Predicates (INEX)

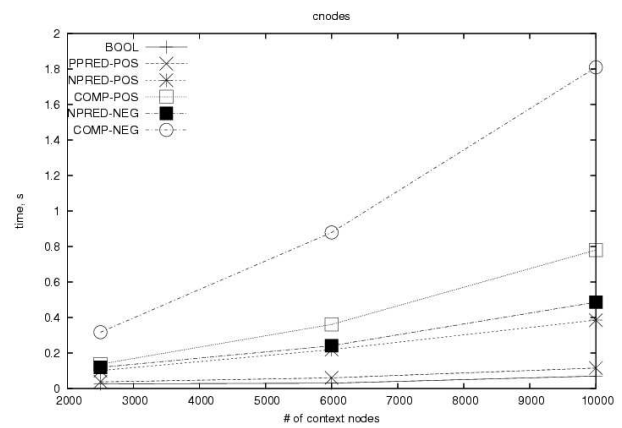

Figure 7: Varying Number Context Nodes (INEX)

lists, thus increasing the number of potential results. Again, PPRED and BOOL are the best, but NPRED also displays only a small increase.

\section{Related Work}

Most IR research [3][31] has focused on methods for relevance estimation and efficient evaluation of keyword queries. In this context, full-text languages have been developed to implement specific primitives, but their formal properties have not been studied. This observation also applies to XML full-text search languages such as XQuery/IR [6], XIRQL [18], XSEarch [15], XRANK [21], XXL [33] and Niagara [37]. In fact, we can represent these languages (see Sections 4.1 and 4.2). Several other works have used relational systems to store inverted lists and translate keyword queries to SQL [11, 17, 22, 29, 30, 37] but they do not study completeness.

Clarke et al. [5] propose a formal model for full-text search with some leverage of structure such as chapters and paragraphs. The model is based on intervals of positions and supports a fixed set of predicates (not) containing, (not) contained in, one of/both of, followed by. Thus, this model is less general than ours and it may be hard to extend it because it is based on intervals of positions. This coarser granularity inherently looses some information since not all positions in an interval may be relevant to the query. No study of expressiveness is provided. 


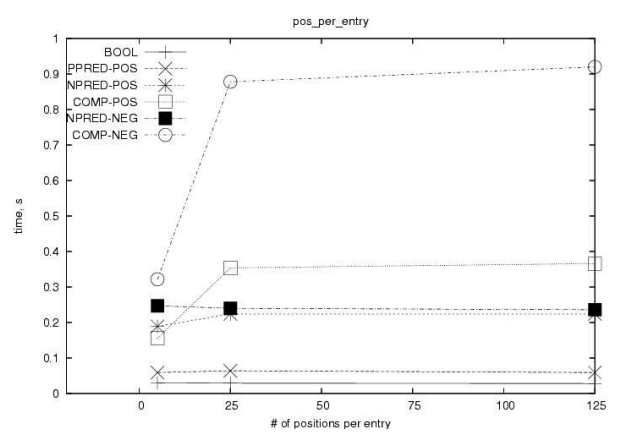

Figure 8: Varying Number of Positions Per Inverted List Entry (INEX)

\section{Conclusion}

We presented a simple, yet powerful formalization of full-text search languages as a basis for studying expressiveness and efficiency. We believe that this work is an important first step for full-text search much like the relational model laid the foundation of extensive database research. We are planning to add new full-text primitives such as stemming, thesaurus and stop-words. We would also like to explore how our formalization in terms of the relational model enables the joint optimization of structured and full-text queries. Finally, we want to study the complexity implications of scoring and top-k techniques [10, 16, 25, 32].

\section{References}

[1] S. Amer-Yahia, C. Botev, J. Robie, J. Shanmugasundaram. TeXQuery: A Full-Text Search Extension to XQuery. http:/www.cs.cornell.edu/database/TeXQuery/.

[2] S. Amer-Yahia, C. Botev, J. Shanmugasundaram. TeXQuery: A Full-Text Search Extension to XQuery. WWW 2004.

[3] R. Baeza-Yates, B. Ribiero-Neto. Modern Information Retrieval. Addison-Wesley, 1999.

[4] G. Bhalotia, A. Hulgeri, C. Nakhe, S. Chakrabarti, S. Sudarshan. Keyword Searching and Browsing in Databases using BANKS. ICDE 2002.

[5] C. Clarke, G. Cormack, F. Burkowski. An Algebra for Structured Text Search and a Framework for its Implementation. Comput. J. 38(1): 43-56 (1995)

[6] J. M. Bremer, M. Gertz. XQuery/IR: Integrating XML Document and Data Retrieval. WebDB 2002.

[7] E. W. Brown. Fast Evaluation of Structured Queries for Information Retrieval. SIGIR 1995.

[8] A. Chandra, P. Merlin Optimal Implementation of Conjunctive Queries in Relational Databases. STOC 1977.

[9] A. Chandra, D. Harel. Structure and Complexity of Relational Queries. FOCS 1980.

[10] S. Chaudhuri, L. Gravano. Evaluating Top-k Selection Queries. In Proc. of 25th VLDB Conf.

[11] T. T. Chinenyanga, N. Kushmerick. Expressive and Efficient Ranked Querying of XML Data. WebDB 2001.

[12] E. F. Codd. A Relational Model of Data for Large Shared Data Banks. Commun. ACM 13(6): $377-387$ (1970).

[13] E.F. Codd. Relational Completeness of Database Sublanguages. In R. Rustin (ed.), Database Systems, PrenticeHall, 1972. 
[14] W.W. Cohen. Integration of Heterogeneous Databases Without Common Domains Using Queries Based on Textual Similarity. SIGMOD 1998.

[15] S. Cohen et al. XSEarch: A Semantic Search Engine for XML. VLDB 2003.

[16] R. Fagin, R. Kumar, D. Sivakumar. Efficient Similarity Search and Classification Via Rank Aggregation. SIGMOD 2003.

[17] D. Florescu, D. Kossmann, I. Manolescu. Integrating Keyword Search into XML Query Processing. WWW 2000.

[18] N. Fuhr, K. Grossjohann. XIRQL: An Extension of XQL for Information Retrieval. SIGIR 2000.

[19] N. Fuhr, T. Rölleke. A Probabilistic Relational Algebra for the Integration of Information Retrieval and Database Systems. ACM TOIS 15(1), 1997.

[20] Y. Hayashi, J. Tomita, G. Kikui. Searching Text-rich XML Documents with Relevance Ranking. SIGIR Workshop on XML and Information Retrieval, 2000.

[21] L. Guo, F. Shao, C. Botev, J. Shanmugasundaram. XRANK: Ranked Keyword Search over XML Documents. SIGMOD 2003.

[22] V. Hristidis, L. Gravano, Y. Papakonstantinou. Efficient IR-Style Keyword Search over Relational Databases. VLDB 2003.

[23] N. Immerman. Relational Queries Computable in Polynomial Time Information and Control. 68(1-3): 86-104 (1986).

[24] Initiative for the Evaluation of XML Retrieval. http://www.is.informatik.uni-duisburg.de/projects/inex03/.

[25] C. Li, K. Chang, I. Ilyas, S. Song. Query Algebra and Optimization for Relational Top-k Queries. SIGMOD 2005

[26] Library of Congress. http://lcweb.loc.gov/crsinfo/xml/.

[27] S.-H. Myaeng, D.-H. Jang, M.-S. Kim, Z.-C. Zhoo. A Flexible Model for Retrieval of SGML Documents. SIGIR 1998.

[28] G. Salton. Automatic Text Processing: The Transformation, Analysis and Retrieval of Information by Computer. Addison Wesley, 1989.

[29] J. Melton, A. Eisenberg. SQL Multimedia and Application Packages (SQL/MM). SIGMOD Record 30(4), 2001.

[30] A. Salminen. A Relational Model for Unstructured Documents. SIGIR 1987.

[31] G. Salton, M. J. McGill. Introduction to Modern Information Retrieval. McGraw-Hill, 1983.

[32] M. Theobald, G. Weikum, R. Schenkel. Top-k Query Evaluation with probabilistic guarantees. In Proc. of VLDB 2004.

[33] A. Theobald, G. Weikum. The Index-Based XXL Search Engine for Querying XML Data with Relevance Ranking. EDBT 2002.

[34] The World Wide Web Consortium. XQuery 1.0: An XML Query Language. W3C Working Draft. http://www.w3.org/TR/xquery/.

[35] The World Wide Web Consortium. XQuery and XPath Full-Text Use Cases. W3C Working Draft. http://www.w3.org/TR/xmlquery-full-text-use-cases/.

[36] M. Vardi. The Complexity of Relational Query Languages. STOC 1982.

[37] C. Zhang, J. Naughton, D. DeWitt, Q. Luo, G. Lohman. On Supporting Containment Queries in Relational Database Management Systems. SIGMOD 2001.

[38] E. Zimanyi. Query Evaluations in Probabilistic Relational Databases. Theoretical Computer Science, 1997. 


\section{A Proofs of Theorems}

\section{Theorem 1: Equivalence of the Full-Text Calculus and Algebra}

Lemma 1. For every full-text algebra expression that only uses position-based predicates from the set Preds, there exists an equivalent full-text calculus expression that only uses position-based predicates from the same set Preds.

Proof Sketch: We will prove that for every algebra expression AlgExpr, which evaluates to a relation $R\left(C N o d e, a t t_{1}, a t t_{2}, \ldots, a t t_{k}\right), k \geq 0$, there exists a calculus query expression $\operatorname{CalcExpr}\left(n, p_{1}, \ldots, p_{k}\right)$ with free variables $\left\{n, p_{1}, \ldots, p_{k}\right\}$, such that $\left\{\left(n, p_{1}, \ldots, p_{k}\right) \mid \operatorname{SearchContext}(n) \wedge \operatorname{hasPos}\left(n, p_{1}\right) \wedge \ldots \wedge\right.$ $\left.h a s P o s\left(n, p_{k}\right) \wedge \operatorname{CalcExpr}\left(n, p_{1}, \ldots, p_{k}\right)\right\}=R$.

The proof is by induction on the structure of AlgExpr.

- If $\operatorname{AlgExpr}=$ SearchContext, then $\operatorname{CalcExpr}(n)=(\exists p \operatorname{hasPos}(n, p) \wedge$ hasPos $(n, p)) \vee$ $\neg(\exists p \operatorname{hasPos}(n, p) \wedge$ hasPos $(n, p))$. CalcExpr $(n)$ is always true; therefore, $\{n \mid \operatorname{SearchContext}(n) \wedge$ CalcExpr $(n)\}$ is equal to the full-text relation SearchContext by its definition.

- If $A l g E x p r=$ HasPos, then CalcExpr $\left(n, p_{1}\right)=$ hasPos $\left(n, p_{1}\right)$, i.e. $\left\{\left(n, p_{1}\right) \mid \operatorname{SearchContext}(n) \wedge\right.$ $\left.h a s P o s\left(n, p_{1}\right)\right\}$ is equal to the full-text relation HasPos by its definition.

- If AlgExpr $=\mathrm{R}_{\mathrm{token}}$, then CalcExpr $(n)=$ hasToken $\left(p,{ }^{\prime}\right.$ token $\left.^{\prime}\right) .\left\{\left(n, p_{1}\right) \mid \operatorname{SearchContext}(n) \wedge\right.$ hasPos $\left(n, p_{1}\right) \wedge$ hasToken $\left(p_{1},{ }^{\prime}\right.$ token $\left.\left.^{\prime}\right)\right\}$ is equal to the full-text relation $R_{\text {token }}$ by its definition.

- If $A l g E x p r=\pi_{\mathrm{CNode}, \mathrm{att}_{1}, \ldots, \text { att }_{\mathrm{i}}}\left(\mathrm{AlgExpr}^{\prime}\right)$, where $A l g E x p r^{\prime}$ is a full-text algebra expression whose equivalent calculus query expression is $\operatorname{CalcExpr}^{\prime}\left(n, p_{1}, \ldots, p_{m}\right)$ and $A l g E x p r^{\prime}$ evaluates to $R^{\prime}\left(C N o d e, a t t_{1}, a t t_{2}, \ldots, a t t_{m}\right), m \geq i$, then $\operatorname{CalcExpr}\left(n, p_{1}, \ldots, p_{i}\right)=\exists p_{i+1} \operatorname{hasPos}\left(n, p_{i+1}\right) \wedge$ $\ldots \exists p_{m}$ hasPos $\left(n, p_{m}\right) \wedge \operatorname{CalcExpr}^{\prime}\left(n, p_{1}, \ldots, p_{m}\right) .\left\{\left(n, p_{1}, \ldots, p_{i}\right) \mid \operatorname{SearchContext}(n) \wedge\right.$ $\bigwedge_{j=1, \ldots, i}$ hasPos $\left.\left(n, p_{j}\right) \wedge \operatorname{CalcExpr}\left(n, p_{1}, \ldots, p_{i}\right)\right\}=\pi_{C N o d e, p_{1}, \ldots, p_{i}}\left\{\left(n, p_{1}, \ldots, p_{m}\right) \mid \operatorname{SearchContext}(n) \wedge\right.$ $\left.\bigwedge_{j=1, \ldots, m} \operatorname{hasPos}\left(n, p_{j}\right) \wedge \operatorname{CalcExpr}^{\prime}\left(n, p_{1}, \ldots, p_{m}\right)\right\}=\pi_{C N o d e, p_{1}, \ldots, p_{i}}\left(R^{\prime}\right)$.

- If AlgExpr $=\mathrm{AlgExpr}_{1} \bowtie \mathrm{AlgExpr}_{2}$, where $A l g E x p r_{1}$ and $A l g E x p r_{2}$ are full-text algebra expressions that evaluate to $R_{i}\left(C N o d e, a t t_{1}, \ldots, a t t_{m_{i}}\right)$ for $i=1,2$, and their equivalent calculus query expressions are $\operatorname{CalcExpr}_{i}\left(n, p_{1}, \ldots, p_{m_{i}}\right)$ for $i=1,2$, then $\operatorname{CalcExpr}\left(n, p_{1}, \ldots, \ldots, p_{m_{1}+m_{2}}\right)=$ $\operatorname{CalcExpr}\left(n, p_{1}, \ldots, p_{m_{1}}\right) \wedge \operatorname{CalcExpr}\left(n, p_{m_{1}+1}, \ldots, p_{m_{1}+m_{2}}\right) .\left\{\left(n, p_{1}, \ldots, p_{m_{1}+m_{2}} \mid \operatorname{SearchContext}(n) \wedge\right.\right.$ $\bigwedge_{j=1, \ldots, m_{1}+m_{2}} \operatorname{hasPos}\left(n, p_{j}\right) \wedge \operatorname{CalcExpr}\left(n, p_{1}, \ldots, p_{m_{1}}\right) \wedge \operatorname{CalcExpr}\left(n, p_{m_{1}+1}, \ldots, p_{m_{1}+m_{2}}\right\}=$ $R_{1} \bowtie R_{2}$.

- If AlgExpr $=\sigma_{\text {pred }\left(\operatorname{att}_{1}, \ldots, \text { att }_{\mathrm{m}}, c_{1}, \ldots, c_{\mathrm{q}}\right)}\left(\operatorname{Expr}^{\prime}\right)$, where $E x p r^{\prime}$ is a full-text algebra expression that evaluates to the relaton $R^{\prime}$ and the equivalent calculus query expression is $\operatorname{CalcExpr}^{\prime}\left(n, p_{1}, \ldots, p_{k}\right)$, then $\operatorname{CalcExpr}\left(n, p_{1}, \ldots, p_{k}\right)=\operatorname{CalcExpr}^{\prime}\left(n, p_{1}, \ldots, p_{k}\right) \wedge \operatorname{pred}\left(\operatorname{att}_{1}, \ldots, \operatorname{att}_{m}, c_{1}, \ldots, c_{q}\right) .\left\{\left(n, p_{1}, \ldots, p_{k}\right) \mid\right.$ $\operatorname{SearchContext}(n) \wedge \bigwedge_{j=1, \ldots, k}$ hasPos $\left(n, p_{j}\right) \wedge \operatorname{CalcExpr}^{\prime}\left(n, p_{1}, \ldots, p_{k}\right) \wedge$ pred $\left(\right.$ att $_{1}, \ldots$, att $\left.\left._{m}, c_{1}, \ldots, c_{q}\right)\right\}=$ $\sigma_{\text {pred }\left(a t t_{1}, \ldots, a t t_{m}, c_{1}, \ldots, c_{q}\right)} R^{\prime}$.

- Let $A l g E x p r=\mathrm{AlgExpr}_{1} \cup \mathrm{AlgExpr}_{2}$, where $A l g \operatorname{Expr}_{1}$ and $A l g \operatorname{Expr}_{2}$ are full-text algebra expressions that evaluate to $R_{i}$ for $i=1,2$ and their equivalent calculus query expressions are $\operatorname{CalcExpr}_{i}\left(n, p_{1}, \ldots, p_{k}\right)$ for $i=1,2$, then $\operatorname{CalcExpr}\left(n, p_{1}, \ldots p_{k}\right)=\operatorname{CalcExpr}_{1}\left(n, p_{1}, \ldots p_{k}\right) \vee$ $\operatorname{CalcExpr}_{2}\left(n, p_{1}, \ldots p_{k}\right) .\left\{\left(n, p_{1}, \ldots, p_{k}\right) \mid \operatorname{SearchContext}(n) \wedge \bigwedge_{j=1, \ldots, k} \operatorname{hasPos}\left(n, p_{j}\right) \wedge\right.$ $\left.\left(\operatorname{CalcExpr}_{1}\left(n, p_{1}, \ldots p_{k}\right) \vee \operatorname{CalcExpr}_{2}\left(n, p_{1}, \ldots p_{k}\right)\right)\right\}=R_{1} \cup R_{2}$. 
- Let $A l g E x p r=A l g E x p r_{1} \cap A l g E x p r_{2}$, where $A l g \operatorname{Expr}_{1}$ and $A l g E x p r_{2}$ are full-text algebra expressions that evaluate to $R_{i}$ for $i=1,2$ and their equivalent calculus query expressions are $\operatorname{CalcExpr}_{i}\left(n, p_{1}, \ldots, p_{k}\right)$ for $i=1,2$, then $\operatorname{CalcExpr}\left(n, p_{1}, \ldots p_{k}\right)=\operatorname{CalcExpr}_{1}\left(n, p_{1}, \ldots p_{k}\right) \wedge$ $\operatorname{CalcExpr}_{2}\left(n, p_{1}, \ldots p_{k}\right) .\left\{\left(n, p_{1}, \ldots, p_{k}\right) \mid \operatorname{SearchContext}(n) \wedge \bigwedge_{j=1, \ldots, k} \operatorname{hasPos}\left(n, p_{j}\right)\right.$ $\left.\wedge\left(\operatorname{CalcExpr}_{1}\left(n, p_{1}, \ldots p_{k}\right) \wedge \operatorname{CalcExpr}_{2}\left(n, p_{1}, \ldots p_{k}\right)\right)\right\}=R_{1} \cap R_{2}$.

- Let AlgExpr $=\mathrm{AlgExpr}_{1}-\mathrm{AlgExpr}_{2}$, where AlgExpr ${ }_{1}$ and AlgExpr ${ }_{2}$ are full-text algebra expressions that evaluate to $R_{i}$ for $i=1,2$ and their equivalent calculus query expressions are $\operatorname{CalcExpr}_{i}\left(n, p_{1}, \ldots, p_{k}\right)$ for $i=1,2$, then $\operatorname{CalcExpr}\left(n, p_{1}, \ldots p_{k}\right)=\operatorname{CalcExpr}_{1}\left(n, p_{1}, \ldots p_{k}\right) \wedge$ $\neg \operatorname{CalcExpr}_{2}\left(n, p_{1}, \ldots p_{k}\right) .\left\{\left(n, p_{1}, \ldots, p_{k}\right) \mid \operatorname{SearchContext}(n) \wedge \bigwedge_{j=1, \ldots, k}\right.$ hasPos $\left(n, p_{j}\right) \wedge$ $\left.\left(\operatorname{CalcExpr}_{1}\left(n, p_{1}, \ldots p_{k}\right) \wedge \neg \operatorname{CalcExpr}_{2}\left(n, p_{1}, \ldots p_{k}\right)\right)\right\}=R_{1}-R_{2}$.

This completes the structural induction. The requirement that full- text algebra queries evaluate to a relation with a single $C N$ ode attribute ensures that the corresponding CalcExpr expression will have only one free variable $-n$. Therefore, $\{n \mid \operatorname{SearchContext}(n) \wedge \operatorname{CalcExpr}(n)\}$ is a valid calculus query.

Lemma 2. For every full-text calculus expression that only uses position-based predicates from the set Preds, there exists an equivalent full-text algebra expression that only uses position-based predicates from the same set Preds.

Proof Sketch: We will prove that for every query calculus expression $\operatorname{CalcExpr}\left(n, p_{1}, \ldots, p_{k}\right)$ with free variables $\left\{n, p_{1}, \ldots, p_{k}\right\}, k \geq 0$, there exists an algebra expression AlgExpr, which evaluates to a relation $R\left(C N o d e, a t t_{1}\right.$, att $_{2}, \ldots$, att $\left._{k}\right)$, such that $\left\{\left(n, p_{1}, \ldots, p_{k}\right) \mid \operatorname{SearchContext}(n) \wedge \bigwedge_{j=1, . ., k} \operatorname{hasPos}\left(n, p_{j}\right) \wedge\right.$ $\left.\operatorname{CalcExpr}\left(n, p_{1}, \ldots, p_{k}\right)\right\}=R$.

The proof is by induction on the structure of CalcExpr.

- If CalcExpr $(n, p)=h a s P o s(n, p)$, then AlgExpr = HasPos. The proof of the equivalence is the same as the analogous case from Lemma 1.

- If $\operatorname{CalcExpr}(n, p)=$ hasToken $\left(p,{ }^{\prime}\right.$ token $\left.^{\prime}\right)$, then $\operatorname{AlgExpr}=\mathrm{R}_{\mathrm{token}}$. The proof of the equivalence is the same as the analogous case from Lemma 1.

- If $\operatorname{CalcExpr}\left(n, p_{1}, \ldots, p_{k}\right)=\operatorname{pred}\left(p_{1}, \ldots, p_{k}, c_{1}, \ldots, c_{q}\right)$, then $\operatorname{AlgExpr}=\sigma_{\mathrm{pred}\left(\mathrm{p}_{1}, \ldots, \mathrm{p}_{\mathrm{k}}, \mathrm{c}_{1}, \ldots, \mathrm{c}_{\mathrm{q}}\right)}$

(HasPos $\bowtie \ldots \bowtie$ HasPos), where the number of joins is $k$. Obviously, $R=\left\{\left(n, p_{1}, \ldots, p_{k}\right) \mid\right.$ $\left.\operatorname{SearchContext}(n) \wedge \bigwedge_{i=1, \ldots, k} h a s P o s\left(n, p_{i}\right) \wedge \operatorname{pred}\left(p_{1}, \ldots, p_{k}, c_{1}, \ldots, c_{q}\right)\right\}$.

- If CalcExpr $\left(n, p_{1}, \ldots, p_{l}, q_{1}^{\prime}, \ldots, q_{m}^{\prime}, q_{1}^{\prime \prime}, \ldots, q_{c}^{\prime \prime}\right)=\operatorname{CalcExpr}_{1}\left(n, p_{1}, \ldots, p_{l}, q_{1}^{\prime}, \ldots, q_{m}^{\prime}\right) \wedge$ $\operatorname{CalcExpr}_{2}\left(n, p_{1}, \ldots, p_{l}, q_{1}^{\prime \prime}, \ldots, q_{c}^{\prime \prime}\right)$, where $k=l+m+c, C_{\text {alcExpr }}$, and CalcExpr $r_{2}$ are calculus query expressions with equivalent algebra expressions $A l g E x p r_{1}$ and $A l g E x p r_{2}$, which evaluate to $R_{1}\left(C N o d e, a t t_{1}, \ldots, a t t_{k}, a t t_{1}^{\prime}, \ldots, a t t_{m}^{\prime}\right)$ and $R_{2}\left(C N o d e, a t t_{1}, \ldots, a t t_{l}, a t t_{1}^{\prime \prime}, \ldots, a t t_{c}^{\prime \prime}\right)$, then AlgExpr $=\left(\right.$ AlgExpr $\left._{1} \bowtie \pi_{\text {CNode }, \mathrm{q}_{1}^{\prime \prime}, \ldots, \mathrm{q}_{\mathrm{c}}^{\prime \prime}} \mathrm{AlgExpr}_{2}\right) \cap\left(\pi_{\mathrm{CNode}, \mathrm{q}_{1}^{\prime}, \ldots, \mathrm{q}_{\mathrm{m}}^{\prime}} \mathrm{AlgExpr}_{1} \bowtie \mathrm{AlgExpr}_{2}\right) . \quad R=$ $\left\{\left(n, p_{1}, \ldots, p_{l}, q_{1}^{\prime}, \ldots, q_{m}^{\prime}, q_{1}^{\prime \prime}, \ldots, q_{c}^{\prime \prime}\right) \mid\left(n, p_{1}, \ldots, p_{l}, q_{1}^{\prime}, \ldots, q_{m}^{\prime}\right) \in R_{1} \wedge\left(n, p_{1}, \ldots, p_{l}, q_{1}^{\prime \prime}, \ldots, q_{c}^{\prime \prime}\right) \in R_{2}\right\}$ $=\left\{\left(n, p_{1}, \ldots, p_{l}, q_{1}^{\prime}, \ldots, q_{m}^{\prime}, q_{1}^{\prime \prime}, \ldots, q_{c}^{\prime \prime}\right) \mid \operatorname{CalcExpr}_{1}\left(n, p_{1}, \ldots, p_{l}, q_{1}^{\prime}, \ldots, q_{m}^{\prime}\right) \wedge\right.$

$\left.\operatorname{CalcExpr}_{2}\left(n, p_{1}, \ldots, p_{l}, q_{1}^{\prime \prime}, \ldots, q_{c}^{\prime \prime}\right)\right\}$, which is exactly what we wnated to show.

- If $\operatorname{CalcExpr}\left(n, p_{1}, \ldots, p_{l}, q_{1}^{\prime}, \ldots, q_{m}^{\prime}, q_{1}^{\prime \prime}, \ldots, q_{c}^{\prime \prime}\right)=\operatorname{CalcExpr}_{1}\left(n, p_{1}, \ldots, p_{l}, q_{1}^{\prime}, \ldots, q_{m}^{\prime}\right) \vee$ $\operatorname{CalcExpr}_{2}\left(n, p_{1}, \ldots, p_{l}, q_{1}^{\prime \prime}, \ldots, q_{c}^{\prime \prime}\right)$, where $k=l+m+c, C$ CalcExpr $_{1}$, and CalcExpr $r_{2}$ are calculus query expressions with equivalent algebra expressions $A l g E x p r_{1}$ and $A l g E x p r_{2}$, which evaluate to $R_{1}\left(C N o d e, a t t_{1}, \ldots, a t t_{k}, a t t_{1}^{\prime}, \ldots, a t t_{m}^{\prime}\right)$ and $R_{2}\left(C N o d e, a t t_{1}, \ldots, a t t_{l}, a t t_{1}^{\prime \prime}, \ldots, a t t_{c}^{\prime \prime}\right)$, then 
AlgExpr $=\left(\right.$ AlgExpr $\left._{1} \bowtie \pi_{\text {CNode }, \mathrm{q}_{1}^{\prime \prime}, \ldots, \mathrm{q}_{\mathrm{c}}^{\prime \prime}} \operatorname{AlgExpr}_{2}\right) \cup\left(\pi_{\mathrm{CNode}, \mathrm{q}_{1}^{\prime}, \ldots, \mathrm{q}_{\mathrm{m}}^{\prime}} \operatorname{AlgExpr}_{1} \bowtie \mathrm{AlgExpr}_{2}\right) . \quad R=$ $\left\{\left(n, p_{1}, \ldots, p_{l}, q_{1}^{\prime}, \ldots, q_{m}^{\prime}, q_{1}^{\prime \prime}, \ldots, q_{c}^{\prime \prime}\right) \mid\left(n, p_{1}, \ldots, p_{l}, q_{1}^{\prime}, \ldots, q_{m}^{\prime}\right) \in R_{1} \vee\left(n, p_{1}, \ldots, p_{l}, q_{1}^{\prime \prime}, \ldots, q_{c}^{\prime \prime}\right) \in R_{2}\right\}$ $=\left\{\left(n, p_{1}, \ldots, p_{l}, q_{1}^{\prime}, \ldots, q_{m}^{\prime}, q_{1}^{\prime \prime}, \ldots, q_{c}^{\prime \prime}\right) \mid \operatorname{CalcExpr}_{1}\left(n, p_{1}, \ldots, p_{l}, q_{1}^{\prime}, \ldots, q_{m}^{\prime}\right) \vee\right.$

$\left.\operatorname{CalcExpr}_{2}\left(n, p_{1}, \ldots, p_{l}, q_{1}^{\prime \prime}, \ldots, q_{c}^{\prime \prime}\right)\right\}$, which is what we wanted to show.

- Let us consirder the case CalcExpr $\left(n, p_{1}, \ldots, p_{k}\right)=\neg C_{a l c E x p r}\left(n, p_{1}, \ldots, p_{k}\right)$, where CalcExpr is a calculus query expression that is equivalent to the algebra expression $A l g E x p r^{\prime}$, which evaluates to $R^{\prime}\left(n, p_{1}, \ldots, p_{k}\right)$. If $k>0$, then $A l g E x p r=($ HasPos $\bowtie \ldots \bowtie$ HasPos $)-A l g E x p r^{\prime}$, where the number of joins is $k . R=\left\{\left(n, p_{1}, \ldots, p_{k}\right) \mid \operatorname{SearchContext}(n) \wedge \bigwedge_{i=1, \ldots, k} \operatorname{hasPos}\left(n, p_{i}\right) \wedge\right.$ $\left.\neg \operatorname{CalcExpr}^{\prime}\left(n, p_{1}, \ldots, p_{k}\right)\right\}$, which is what we wanted to show.

If $k=0$, then AlgExpr $=$ SearchContext - AlgExpr $^{\prime}$ and $R=\{n \mid \operatorname{SearchContext~}(n) \wedge$ SearchContext $\left.(n) \wedge \neg C_{\text {alcExpr }}(n)\right\}$

- If CalcExpr $\left(n, p_{1}, \ldots, p_{k}\right)=\exists p_{k+1}$ hasNode $\left(n, p_{k+1}\right) \wedge \operatorname{CalcExpr}^{\prime}\left(n, p_{1}, \ldots, p_{k+1}\right)$, where CalcExpr is a calculus query expression that is equivalent to the algebra expression $A l g E x p r^{\prime}$, which evaluates to $R^{\prime}$, then AlgExpr $=\pi_{\mathrm{CNode}, \mathrm{p}_{1}, \ldots, \mathrm{p}_{\mathrm{k}}} \mathrm{R}^{\prime}$ and $R=\left\{\left(n, p_{1}, \ldots, p_{k}\right) \mid \operatorname{SearchContext}(n) \wedge\right.$ $\left.\exists p_{k+1}\left(n, p_{1}, \ldots, p_{k+1}\right) \in R^{\prime}\right\}=\left\{\left(n, p_{1}, \ldots, p_{k}\right) \mid \operatorname{SearchContext}(n) \wedge \exists p_{k+1} \operatorname{CalcExpr}^{\prime}\left(n, p_{1}, \ldots, p_{k+1}\right)\right\}$.

- Let $\operatorname{CalcExpr}\left(n, p_{1}, \ldots, p_{k}\right)=\forall p_{k+1} \operatorname{hasNode}\left(n, p_{k+1}\right) \Rightarrow \operatorname{CalcExpr}^{\prime}\left(n, p_{1}, \ldots, p_{k+1}\right)$, where CalcExpr $r^{\prime}$ is a calculus query expression that is equivalent to the algebra expression AlgExpr'. We use the equation $\operatorname{CalcExpr}\left(n, p_{1}, \ldots, p_{k}\right)=\neg \exists p_{k+1} \neg \operatorname{CalcExpr}^{\prime}\left(n, p_{1}, \ldots, p_{k+1}\right)$ and apply the previous case..

For every calculus query, its query expression has only one free variable, $n$, therefore the equivalent algebra query evaluates to a relation that contains a single column, $C N o d e$. Therfore, it is a valid algebra query.

The above two Leammas prove the equivalence of the full-text calculus and algebra.

\section{Theorem 4: Completeness of BOOL when $\mathcal{T}$ is finite}

Proof Sketch: Let $F=\{n \mid$ SearchContext $(n) \wedge P(n)\}$ be a calculus query expression. We will prove that there exists an equivalent Query expression $E$ in BOOL. Without loss of generality, we assume that every quantified variable in $F$ has a unique name. Let these position variable names be $p_{1}, p_{2}, \ldots, p_{m}$.

We first normalize $P(n)$ using the sequence of equivalence transformations presented below.

1. (Sink Negations) Move all negations down to the predicates hasPos $\left(n, p_{i}\right)$ and hasToken $\left(p_{i}, t\right)$. Replace any repetitive negations $\neg \neg A$ with $A$. Invert quantifiers: $\neg \exists p h a s P o s(n, p) \wedge A$ is replaced with $\forall p$ hasPos $(n, p) \Rightarrow \neg A$ and $\neg \forall p$ hasPos $(n, p) \Rightarrow A$ is replaced with $\exists p$ hasPos $(n, p) \wedge \neg A$.

2. (Group) Move every expression of the form hasToken $\left(p_{i}, t\right)$ and $\neg$ hasToken $\left(p_{i}, t\right)$ out of the scope of any quantifier over a variable different from $p_{i}$. This is possible because hasToken is applied on only one position variable. Formally, the transformation is a repeated application of $\mathcal{Q} p_{j} A \circ B \mapsto$ $B \circ \mathcal{Q} p_{j} A$ where $\mathcal{Q} \in\{\exists, \forall\}, \circ \in\{\wedge, \vee\}$, and $B$ has no free variable $p_{j}$. Use the commutativity of $\wedge$ and $\vee$ to group the above predicate expressions next to each other and right after hasPos $\left(n, p_{i}\right)$. We get a propositional formula with propositions of the form $\mathcal{Q}_{i} p_{i} A_{i}\left(n, p_{i}\right)$ where $\mathcal{Q}_{i} \in\{\exists, \forall\}$.

3. (Remove universal quantification) Remove any universal quantifers by replacing $\forall p_{i}$ hasPos $\left(n, p_{i}\right) \Rightarrow$ $X$ with $\neg \exists p_{i} h a s P o s\left(n, p_{i}\right) \wedge \neg X$. We get a propositional formula over propositions of the form $\exists p_{i} \operatorname{hasPos}\left(n, p_{i}\right) \wedge B_{i}\left(n, p_{i}\right)$. 
4. (Local DNF) Convert each $B_{i}\left(n, p_{i}\right)$ to DNF.

5. (Split) Replace $\exists p$ hasPos $(n, p) \wedge(X(n, p) \vee Y(n, p))$ with $\left(\exists p^{\prime} \operatorname{hasPos}\left(n, p^{\prime}\right) \wedge X\left(n, p^{\prime}\right)\right) \vee$ $\left(\exists p^{\prime \prime} h a s P o s\left(n, p^{\prime \prime}\right) \wedge Y\left(n, p^{\prime \prime}\right)\right)$ to $\exists p_{i} h a s P o s\left(n, p_{i}\right) \wedge B_{i}\left(n, p_{i}\right)$ for every disjunct in $B_{i}\left(n, p_{i}\right)$. Let the new position variables be $q_{1}, \ldots, q_{k}$. We get a propositional formula over propositions of the form $\exists q_{j}$ hasPos $\left(n, q_{j}\right) \wedge C_{j}\left(n, q_{j}\right)$ where $C_{j}$ is a conjunction.

6. (Global DNF) Consider $F$ to be a propositional formula over propositions of the form $\exists q_{j}$ hasPos $\left(n, q_{j}\right) \wedge$ $C_{j}\left(n, q_{j}\right)$. Convert it to a DNF.

We define $Q E(F)$ for a calculus query expression $F$ as the equivalent query in BOOL.

We observe that after the normalization $F=\left\{n \mid \operatorname{SearchContext}(n) \wedge\left(\bigvee_{i} \wedge_{j} D_{i, j}\right)\right\}=\bigcup_{i} \bigcap_{j}\{n \mid$ SearchContext $\left.(n) \wedge D_{i, j}\right\}$, where each $D_{i, j}$ is either of the form $\exists q \operatorname{hasPos}(n, p) \wedge C(n, q)$ or of the form $\neg \exists q$ hasPos $(n, p) \wedge C(n, q)$, as in step GlobalDNF from the normalization. Therefore, $F$ can be decomposed into the calculus expressions $F_{i_{j}}=\left\{n \mid \operatorname{SearchContext}(n) \wedge D_{i_{j}}\right\}$ and it is not difficult to see that $Q E(F)=\left(Q E\left(F_{1,1}\right)\right.$ AND ... AND $\left.Q E\left(F_{1, r_{1}}\right)\right)$ OR ... OR $\left(Q E\left(F_{s, 1}\right)\right.$ AND ... AND QE $\left.\left(F_{s, r_{s}}\right)\right)$. Thus, we can focus only on converting each $F_{i, j}$.

As seen above, each $F_{i, j}$ is of the form $\exists p \operatorname{hasPos}(n, p) \wedge \bigwedge_{r} H_{r}(n, p)$ or of the form $\neg \exists p$ hasPos $(n, p) \wedge$ $\bigwedge_{r} H_{r}(n, p)$, where $H_{r}(n, p)$ is hasToken $(p, t)$ or $\neg$ hasToken $(p, t)$. In either case, we can consider there are no duplicates among $H_{r}(n, p)$; otherwise, we can simply eliminate them.

Let us first consider the case where $F_{i, j}=\exists \operatorname{hasPos}(n, p) \wedge \bigwedge_{t} H_{t}(n, p)$.

- If there exists $r_{1}$ and $r_{2}$ such that $H_{r_{1}}(n, p)=\operatorname{hasToken}\left(p, t_{1}\right), H_{r_{2}}(n, p)=\operatorname{hasToken}\left(p, t_{2}\right)$, and $t_{1} \neq t_{2}$, then the condition "one token per position" (Section 2.2) is violated. Therefore, $F_{i, j}$ is the empty set. $Q E\left(F_{i, j}\right)=$ ANY AND NOT $\left.\left(t_{1} O R \ldots O R t_{c}\right)\right)$, where $\mathcal{T}=\left\{t_{1}, \ldots, t_{c}\right\}$ is the set of all tokens. Intuitively, this query returns the empty set because it requires the result nodes to contain a token that is not in $\mathcal{T}$, which is impossible.

- If there exists $r_{1}$ and $r_{2}$ such that $H_{r_{1}}(n, p)=\operatorname{hasToken}(p, t)$ and $H_{r_{2}}(n, p)=\neg$ hasToken $(p, t)$ then this is an obvious contradiction and $F_{i, j}$ is the empty set. We define $Q E\left(F_{i, j}\right)=$ ANY AND NOT $\left.\left(t_{1} O R \ldots O R t_{c}\right)\right)$ as above.

- Let there exists $r_{1}$ and there does not exist $r_{2}$ such that $H_{r_{1}}(n, p)=$ hasToken $(p, t)$ and $H_{r_{2}}(n, p)=$ $\neg$ hasToken $(p, t)$. Then we can ignore any $H_{r}(n, p)$ which contains $\neg$ hasToken $\left(p, t^{\prime}\right)$ for some token $t^{\prime} \neq t$. The latter are trivially satisfied. In this case, $F_{i, j}=\{n \mid \operatorname{SearchContext}(n) \wedge$ $\exists p \operatorname{hasPos}(n, p) \wedge$ hasToken $(p, t)\}$, which is exactly the semantics for $Q E\left(F_{i, j}\right)=t$.

- The last case is $F_{i, j}=\left\{n \mid \operatorname{SearchContext}(n) \wedge \exists p \operatorname{hasPos}(n, p) \wedge \neg \operatorname{hasToken}\left(p, t_{i_{1}}\right) \wedge\right.$ $\ldots \wedge \neg$ hasToken $\left(p, t_{i_{v}}\right)$. This expression can be interpreted as the condition that $n$ contains a token from the complement $\left\{t_{j_{1}}, \ldots, t_{j_{u}}\right\}$ of $\left\{t_{i_{1}}, \ldots, t_{i_{v}}\right\}$ with regards to the set $\mathcal{T}:\left\{t_{j_{1}}, \ldots, t_{j_{u}}\right\}=$ $\mathcal{T}-\left\{t_{i_{1}}, \ldots, t_{i_{v}}\right\}$. Due to the finiteness of $\mathcal{T}, F_{i, j}=\{n \mid \operatorname{SearchContext}(n) \wedge \exists p \operatorname{hasPos}(n, p) \wedge$ $\left(\operatorname{hasToken}\left(p, t_{j_{1}}\right) \vee \ldots \vee\right.$ hasToken $\left.\left.\left(p, t_{j_{u}}\right)\right)\right\}=\bigcup_{r}\{n \mid \operatorname{SearchContext}(n) \wedge \exists p \operatorname{hasPos}(n, p) \wedge$ $\left.\operatorname{hasToken}\left(p, t_{j_{r}}\right)\right\}$. The latter is trivially equivalent to the query $Q E\left(F_{i, j}\right)=t_{j_{1}}$ OR $\ldots \quad$ OR $t_{j_{u}}$.

In case $F_{i, j}=\neg \exists p$ hasPos $(n, p) \wedge \bigwedge_{t} H_{t}(n, p)$, then $Q E\left(F_{i, j}\right)=$ NOT $Q E\left(\neg F_{i, j}\right)$, where $\neg F_{i, j}$ is transformed as in the previous case. 


\section{Theorem 6: Completeness of COMP}

Proof Sketch: We will prove that every calculus query can be represented by a COMP query CompQuery. We use induction on the structure of the query expression $\operatorname{CalcExpr}\left(n, p_{1}, \ldots, p_{k}\right)$.

- If CalcExpr $(n, p)=$ hasPos $(n, p)$, then CompQuery $=p$ HAS ANY. This is equivalent to CalcExpr by definition.

- If CalcExpr $(n, p)=$ hasToken $\left(p,{ }^{\prime}\right.$ token $\left.^{\prime}\right)$, then CompQuery $=p$ HAS 'token'. This is equivalent to CalcExpr by definition.

- If CalcExpr $\left(n, p_{1}, \ldots, p_{k}\right)=\operatorname{pred}\left(p_{1}, \ldots, p_{k}, c_{1}, \ldots, c_{q}\right)$, then CompQuery $=\operatorname{pred}\left(\mathrm{p}_{1}, \ldots, \mathrm{p}_{\mathrm{k}}, \mathrm{c}_{1}, \ldots, \mathrm{c}_{\mathrm{q}}\right)$. This is equivalent to CalcExpr by definition.

- If $\operatorname{CalcExpr}\left(n, p_{1}, \ldots, p_{l}, q_{1}^{\prime}, \ldots, q_{m}^{\prime}, q_{1}^{\prime \prime}, \ldots, q_{c}^{\prime \prime}\right)=\operatorname{CalcExp}_{1}\left(n, p_{1}, \ldots, p_{l}, q_{1}^{\prime}, \ldots, q_{m}^{\prime}\right) \wedge$

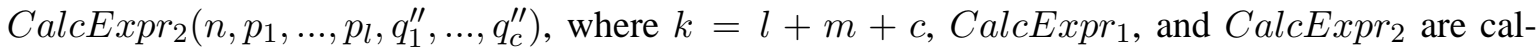
culus query expressions with equivalent COMP queries be CompQuery $y_{1}$ and CompQuery Con $_{2}$, then CompQuery $=$ CompQuery $y_{1}$ AND CompQuery $y_{2}$. This is equivalent to CalcExpr by definition.

- If $\operatorname{CalcExpr}\left(n, p_{1}, \ldots, p_{l}, q_{1}^{\prime}, \ldots, q_{m}^{\prime}, q_{1}^{\prime \prime}, \ldots, q_{c}^{\prime \prime}\right)=\operatorname{CalcExp}_{1}\left(n, p_{1}, \ldots, p_{l}, q_{1}^{\prime}, \ldots, q_{m}^{\prime}\right) \vee$ $\operatorname{CalcExpr}_{2}\left(n, p_{1}, \ldots, p_{l}, q_{1}^{\prime \prime}, \ldots, q_{c}^{\prime \prime}\right)$, where $k=l+m+c, C_{\text {alcExpr }}$, and CalcExpr $r_{2}$ are calculus query expressions with equivalent COMP queries be CompQuery $y_{1}$ and CompQuery ${ }_{2}$, then CompQuery $=$ CompQuery $_{1}$ OR CompQuery 2 . This is equivalent to CalcExpr by definition.

- If $\operatorname{CalcExpr}\left(n, p_{1}, \ldots, p_{k}\right)=\neg \operatorname{CalcExpr}^{\prime}\left(n, p_{1}, \ldots, p_{k}\right)$, where $C a l c E x p r^{\prime}$ is a calculus query expression that is equivalent to the COMP query CompQuery', then CompQuery=NOT CompQuery'. This is equivalent to CalcExpr by definition.

- If CalcExpr $\left(n, p_{1}, \ldots, p_{k}\right)=\exists p_{k+1}$ hasNode $\left(n, p_{k+1}\right) \wedge \operatorname{CalcExpr}^{\prime}\left(n, p_{1}, \ldots, p_{k+1}\right)$, where CalcExpr' is a calculus query expression that is equivalent to the COMP query CompQuery', then CompQuery $=\mathrm{SOME} p_{k+1}$ (CompQuery $\left.{ }^{\prime}\right)$. This is equivalent to CalcExpr by definition.

- If $\operatorname{CalcExpr}\left(n, p_{1}, \ldots, p_{k}\right)=\forall p_{k+1} \operatorname{hasNode}\left(n, p_{k+1}\right) \Rightarrow \operatorname{CalcExpr}^{\prime}\left(n, p_{1}, \ldots, p_{k+1}\right)$, where CalcExpr' is a calculus query expression that is equivalent to the COMP query CompQuery ${ }^{\prime}$, then CompQuery = EVERY $p_{k+1} \quad\left(\right.$ CompQuery $\left.{ }^{\prime}\right)$. This is equivalent to CalcExpr by definition. 